\title{
Spring Barley Resistance and Tolerance to the Cereal Cyst Nematode Heterodera avenae
}

Juliet M., Marshall, University of Idaho, Cereals Research and Extension Program, Idaho Falls 83404; and Richard W. Smiley, Oregon State University, Columbia Basin Agricultural Research Center, Pendleton 97801

\begin{abstract}
Marshall, J. M., and Smiley, R. W. 2016. Spring barley resistance and tolerance to the cereal cyst nematode Heterodera avenae. Plant Dis. 100:396-407.

Heterodera avenae is a cereal cyst nematode that reduces wheat yields in the Pacific Northwest of the United States. Barley is also susceptible but there were no previous reports of resistance or tolerance to $H$. avenae in the United States. Spring barley cultivars were assayed in H. avenae-infested fields over 2 years. Cultivars were planted in plots treated or not treated with aldicarb. Forty-five cultivars were evaluated for the market classes of two- and six-row feed barley cultivars and two- and six-row malt barley cultivars. One two-row feed barley ('Lenetah') was ranked as resistant and four were tolerant or very tolerant. In total, 1 two-row malt barley ('Odyssey') was very resistant

and 10 were tolerant or very tolerant. Two six-row feed and two sixrow malt barley cultivars were tolerant or very tolerant but none were resistant. Seven feed barley cultivars were ranked as having a balance of at least moderate resistance plus moderate tolerance: 'Champion', Lenetah, 'Xena', 'Idagold II', 'Transit', 'Millennium', and 'Goldeneye'. This is the first report of resistance and tolerance of barley in H. avenae-infested fields in the Pacific Northwest. Barley productivity can be improved by planting resistant plus tolerant cultivars or by using highly resistant and highly tolerant cultivars as parents in barley improvement programs.
\end{abstract}

Barley (Hordeum vulgare L.) is harvested from about 300,000 ha annually in the Pacific Northwest (PNW), with 22, 5, and $1 \%$ harvested from the states of Idaho, Washington, and Oregon, respectively (USDA-NASS 2013). The cereal cyst nematode Heterodera avenae Woll. reduces grain yield of wheat in localized regions of each of these states (Smiley 2009; Smiley et al. 1994, 2005b, 2011a, 2012, 2013). An initial density of more than 3,000 H. avenae eggs plus juveniles per $\mathrm{kg}$ of soil is generally capable of reducing yields of wheat, barley, oat, and rye (Andersson 1982).

Rotation of cereal crop hosts of $H$. avenae with 2 years of nonhost broadleaf crop species or fallow have long been recommended to reduce the density of this nematode on highly infested fields (Andersson 1982; Fisher and Hancock 1991; Rivoal and Sarr 1987; Smiley et al. 1994; Tikhonova et al. 1975). The 3-year rotation for this purpose alone is seldom profitable in the PNW and is generally not an accepted practice in regions where 2-year rotations of a cereal crop and potato or a cereal crop and a pulse crop are most profitable in fields where $H$. avenae is absent or at low density. No chemical or biological nematicides are currently available to manage H. avenae (Smiley et al. 2012).

Resistance is characterized by cultivars that greatly suppress or prevent reproduction of nematodes (Cook and Evans 1987). Resistance to $H$. avenae in barley was first discovered in 1920 and was characterized in 1961 (Andersen 1961). Resistance of barley to $H$. avenae has been a specific crop improvement objective in other countries for more than five decades (Andersen 1961; Andersen and Andersen 1973; Cotten 1967; Holgado et al. 2009; Nielsen 1982; O'Brien and Fisher 1974; Valocká et al. 1994; Williams 1970). The best currently characterized sources of resistance to $H$. avenae in barley are the genes mapped to the Ha2 locus on chromosome $2 \mathrm{H}$ (Kretschmer et al. 1997) and to the Ha4 locus mapped

Corresponding author: R. Smiley; E-mail: richard.smiley@oregonstate.edu

*The $\boldsymbol{e}$-Xtra logo stands for "electronic extra" and indicates that one supplementary figure and two supplementary tables are published online.

Accepted for publication 29 June 2015.

http://dx.doi.org/10.1094/PDIS-05-15-0498-RE

(C) 2016 The American Phytopathological Society on chromosome 5H (Barr et al. 1998). According to the gene nomenclature system of Moseman (1972), these gene loci should be designated as Rha2 and Rha4, respectively. However, except for 12 noncommercial barley entries in an international screening array to identify pathotypes of $H$. avenae, which were evaluated under controlled-environment conditions (Smiley et al. 2011b), we are not aware of any contemporary barley cultivars or breeding lines having been evaluated for resistance to $H$. avenae in the United States. There are apparently no commercial barley cultivars in the United States for which the registration includes a report of resistance to H. avenae (USDA-ARS-GRIN 2015).

Barley is generally more tolerant to invasion by $H$. avenae than are oat and wheat (Andersson 1982; O'Brien and Fisher 1977) but tolerance also has not been evaluated in North America. Tolerant cultivars are characterized as having an ability to withstand or recover from nematode invasion and to yield well in comparison with noninvaded plants (Cook and Evans 1987; Fisher 1982). Tolerance is usually estimated in the field by comparing the yield of a specific plant cultivar in a naturally infested soil that is either left untreated or is treated with a nematicide such as aldicarb to reduce the impact of the existing nematode population (Brown 1987; Meagher et al. 1978; Smiley 2009; Taylor et al. 1999).

Roots of both resistant and tolerant cultivars are invaded by $H$. avenae, which may cause an intolerant reaction in some cultivars before the resistance trait becomes expressed in resistant cultivars (O'Brien and Fisher 1977; Ogbonnaya et al. 2001; Oka et al. 1997). This contributes to the inability of some resistant cultivars to produce grain yields that are competitive with more susceptible cultivars to which they are being compared (Wilson et al. 1983). Growers are often reluctant to plant resistant cultivars that produce yields that are lower than some susceptible cultivars (Rivoal and Cook 1993). Therefore, cultivars with both resistance and tolerance are required for both optimal yield performance in existing plantings and reducing the future risk to subsequent plantings of intolerant cultivars or crops (Andersen 1961; Brown 1987; Fisher 1982). Because barley is generally more tolerant of $H$. avenae than are other cereals, it has been predicted that there is a greater potential in barley than in other cereal species to identify cultivars that are both resistant to and tolerant of this nematode (Andersson 1982; O'Brien and Fisher 1977).

The objective of this research was to perform the first evaluation of barley cultivars for tolerance and resistance to $H$. avenae in the United States. We evaluated 45 spring barley cultivars in four market 
classes (two-row feed, two-row malt, six-row feed, and six-row malt) over a 2-year period in naturally infested fields to examine our hypotheses that North American barley cultivars differ in tolerance and resistance, and that cultivars with acceptable balances of both resistance and tolerance could be identified.

\section{Materials and Methods}

Location. Trials during 2013 and 2014 were performed on fields on a farm near St. Anthony in Fremont County, Idaho (latitude 43.922 N, longitude $-111.638 \mathrm{~W}$, and altitude of $1,521 \mathrm{~m}$ ). The climate is characterized as a semiarid continental type with cold winters and warm, dry summers. The mean annual precipitation is $352 \mathrm{~mm}$ and the soil is a St. Anthony gravelly sandy loam. Supplemental water was applied to each field by sprinkler irrigation. Fields were selected based upon a previous knowledge that they were infested by $H$. avenae. In each field, the spring barley trials were performed during the spring grain cycle of a 2-year rotation of potato and a spring cereal. Soil was cultivated and seed was planted as soon as possible after the soil thawed and became friable after the winter freeze. Cultivation by deep disking (20-cm depth) was performed during the autumn following the potato crop and a light rotovating (10-cm depth) was performed to prepare a uniform surface prior to planting the spring barley. Preplant density of plant-parasitic nematode genera was determined at the time of planting, and the processing procedure for preplant and postharvest soil samples is described later.

Experimental design. Three spring barley experiments were planted at St. Anthony, ID on 18 April 2013. Experiment number 1 included 16 cultivars of two-row feed barley. Experiment number 2 included 19 cultivars of two-row malting barley, and experiment number 3 included 10 cultivars of six-row barley that included four feed and six malting barley cultivars. Cultivars for each trial were those that were evaluated for agronomic traits, grain yield, grain quality, and foliar diseases at four other locations in southeast and southcentral Idaho (Marshall et al. 2014).

The three experimental blocks were planted end to end without a border area between trials. Each trial consisted of four replicates of each barley entry planted into a split-plot design. Each plot consisted of two drill rows in a 0.9-by-9-m area. Cultivars were randomized within each replicate (main plot) and each cultivar was split (subplots) into an adjacent nematicide-treated and control plot. The field had been previously determined to be infested rather uniformly with $H$. avenae and, therefore, the preplant estimation of nematode density consisted of a single composite soil sample from the total area encompassed by the three experiments. The composite sample consisted of 25 soil cores ( $2.5 \mathrm{~cm}$ in diameter) collected to $30-\mathrm{cm}$ depth.

The three experiments were repeated during 2014. Entries and experimental designs were identical except that the trials were separated by about $20 \mathrm{~m}$ from one another within a field in which the $H$. avenae density was found to be more aggregately distributed than in the field used during 2013. Therefore, preplant sampling for nematode density was taken individually from each of the three experimental blocks. Planting was performed on 10 April 2014.

Experimental procedures. A locally fabricated no-till drill was used to plant each trial. The drill was equipped with a cone-seeder, two Gandy distributors, and four series of row openers spaced at $36 \mathrm{~cm}$. Fluted opening coulters were mounted on a front tool bar and were followed by a sweep-type deep bander for dispensing fertilizer. A second toolbar was used to mount double-disk openers to dispense seed in line with the opening coulter and deep bander. One Gandy distributor was used to dispense fertilizer $5 \mathrm{~cm}$ below and $4 \mathrm{~cm}$ to each side of the seed row. Seed was dispensed through a cone-seeder and placed into moist soil at $4.0-$ and $2.5-\mathrm{cm}$ depths during 2013 and 2014, respectively. Seed was planted at a density of $205 \mathrm{seed} / \mathrm{m}^{2}$.

Seed was planted with or without application of the nematicide aldicarb (Temik 15G; Bayer CropScience, Research Triangle Park, $\mathrm{NC}$ ), with aldicarb at the rate of $4.2 \mathrm{~kg} / \mathrm{ha}$. Aldicarb was metered from a Gandy distributor on the drill and was placed with the seed into two rows on one side of the seed drill. Untreated controls and aldicarb treatments (subplots) each consisted of two rows to provide side-by-side comparisons of varietal performance in replicated treated and untreated plots.

Fertilizer was banded uniformly under all four seed rows at the time of planting, with $\mathrm{N}$ at a rate of $123 \mathrm{~kg} / \mathrm{ha}$ formulated as a $1: 1$ blend of 16-20-0 and 46-0-0. During 2013, seed was treated with difenoconazole plus mefenoxam at 120 and $30 \mathrm{mg}$, respectively, of active ingredient (a.i.) per kilogram of seed (Dividend Extreme; Syngenta Crop Protection, Greensboro, NC). During 2014, the seed was treated with the fungicides difenoconazole, mefenoxam, and ipconazole and the insecticide thiamethoxam at 180, 44, 15, and $129 \mathrm{mg}$ a.i./kg of seed, respectively (Dividend Extreme + Rancona 3.8FS + Cruiser 5FS, formulated as Cruiser Maxx Custom Blend; Pendleton Grain Growers, Pendleton, OR).

Weed control consisted of a preplant application of glyphosate and a postemergence application of a mixture of pyrasulfotole, bromoxynil, fluroxypyr, and florasulam (Huskie; Bayer CropScience) mixed with Starane Flex (Dow AgroSciences, Indianapolis, IN). During 2013, stripe rust was prevented by application of propiconazole plus trifloxystrobin (Stratego; Bayer CropScience) mixed with the herbicides.

The timing of plant sampling to determine resistance was based upon plant growth stages predicted using the online barley phenology model Barley R Miller MSU (http://uspest.org/cgi-bin/ddmodel.us ). The model is based upon accumulation of GGD expressed using $0^{\circ} \mathrm{C}$ as the base temperature at which no heat accumulation or plant growth is calculated. We used data from a site located close to our experiment: Saint Anthony 1 WNW AM ID. Calculations are initiated when the seed is planted and the predicted date of coleoptile emergence occurs at 144 GGD after barley seed begins to imbibe water. The Miller phenological model for barley predicts that anthesis will occur at the time that 967 growing degree days (GDD) have accumulated after the date of planting, and is when females swollen with eggs are most visible.

The development of a closely related species, $H$. filipjevi, is best fitted to a base temperature of $8^{\circ} \mathrm{C}$ (Hajihassani et al. 2010). Yuan et al. (2014) determined that the invasion and development dynamics of $H$. avenae and $H$. filipjevi are almost the same. GGD calculations for development of these nematodes begins when roots become invaded, which can occur as soon as roots begin to appear (Kerry and Jenkinson 1976). Root establishment can be approximated to the time of coleoptile emergence. Juveniles in the soil matrix retain their ability to invade roots for many weeks (Davies and Fisher 1976). White females of $H$. filipjevi start to become visible at about $209 \mathrm{GDD}\left(8^{\circ} \mathrm{C}\right.$ base $)$ and eggs become embryonated in fully swollen white females at about 358 GDD (Hajihassani et al. 2010). Therefore, the timing of sampling for $H$. avenae differs somewhat from the barley phenological model parameters stated above.

For this study, we collected roots of 10 plants from each of three replicates of each treatment when more than 500 GDD had accumulated after seedling emergence, which occurred 20 days after fully embryonated eggs were predicted and 15 days after anthesis, on 13 June 2013 and 23 July 2014. Roots were used to examine the incidence and severity of the classical root-knotting symptoms, in which adventitious roots proliferate at points on the root axis where the nematode established a specialized feeding cell. Roots were dug to a depth of about $15 \mathrm{~cm}$ from three locations within each of the plots examined. Roots were washed and were rated visually for incidence and severity. Incidence was calculated as the percentage of plants exhibiting at least one knotted root. The severity scale was as follows: $1=$ no evidence of damage, $2=1$ to 3 knots/root system, $3=$ 3 to 5 knots, $4=>5$ knots and $<20 \%$ reduction in plant height or root mass, and $5=>5$ knots and $>20 \%$ reduction in plant height or root mass.

Resistance was measured by counting the number of $H$. avenae swollen white females on roots of five plants per plot by manually rubbing roots to dislodge the white females from washed roots. The swollen females were collected on a 60-mesh sieve and were washed onto filter papers and counted with the aid of a dissecting microscope. Cultivars were rated as very resistant $(\leq 1$ swollen female/plant), resistant (1.1 to 3), moderately resistant (3.1 to 6), 
moderately susceptible (6.1 to 12 ), susceptible 12.1 to 25 ), or very susceptible $(>25)$.

Grain yield and test weight were calculated by harvesting two-row plots of all four replicates using a Wintersteiger plot combine (Wintersteiger Inc., Salt Lake City, UT) on 20 August 2013 and 9 September 2014. Tolerance ratings were assigned to cultivars according to the scale used previously by Smiley et al. (2013): very tolerant ( $\leq 5 \%$ yield increase with nematicide), tolerant (5.1 to $10 \%$ ), moderately tolerant (10.1 to $15 \%)$, moderately intolerant (15.1 to $30 \%)$, intolerant (30.1 to $50 \%)$, or very intolerant $(>50.1 \%)$.

A primary objective of this research was to identify cultivars that failed to meet criteria for full resistance or tolerance but exhibited an acceptable balance among those traits. Data grouped over 2 years were used to establish a ranking of cultivars that exhibited at least a moderate level of resistance ( $\leq 6 \%$ swollen females/plant) plus at least a moderate level of tolerance ( $\leq 15 \%$ yield increase with nematicide). A second measure of resistance was performed by determining nematode density in soil samples collected after grain harvest. Samples during 2013 were taken using manually operated soil probes and consisted of 20 soil cores ( $2.5 \mathrm{~cm}$ in diameter by $30 \mathrm{~cm}$ in depth) composited from each plot. Cores were taken directly below rows of stubble from three replicates of each treatment sampled. For the tworow feed barley, five cultivars were sampled in both untreated and nematicide-treated plots. Six cultivars were examined for the tworow malt barley and the six-row barley. Two cultivars from the nematicide-treated plots were selected to represent those for which we had found a high number of white females on roots in untreated plots, for two cultivars that had a low number, and for two cultivars of special interest to local growers. During 2014, postharvest sampling for nematode density was from three replicates of all cultivars in the control treatment and two cultivars in the nematicide treatment. Due to dry soil conditions, postharvest sampling during 2014 consisted of cores being collected using a tractor-mounted Giddings GSTRS Hydraulic Soil Sampler (Giddings Machine Company, Windsor, CO) with a slotted soil tube $5 \mathrm{~cm}$ in diameter and $150 \mathrm{~cm}$ long. Two cores separated by $2 \mathrm{~m}$ were collected to $30-\mathrm{cm}$ depth in each plot. Soil from the two cores was composited into a single sample for each plot.

Nematode density and identification. All preplant and postharvest soil samples were submitted to Western Laboratories (Parma, ID) for extraction and enumeration of nematodes. The lab uses a modified Oosterbrink elutriator extraction method, described in greater detail by Smiley et al. (2011a). Briefly, vermiform and encysted life stages were extracted and collected on separate sieves. Cysts were broken mechanically to extract eggs and larvae and the suspension was added to the suspension of vermiform life stages present in the soil. The suspension was then concentrated through multiple sequences of centrifugation and density flotation using a magnesium sulfate solution. Western Laboratories reported numbers of plant-parasitic nematodes that were identified to the genus level. These methods were used to calculate the density of $H$. avenae eggs plus juveniles per kilogram of soil in each sample.

Heterodera spp. extracted from fields where the trials were established during the 2 years were identified as $H$. avenae during previous research (Smiley et al. 2011b; Yan and Smiley 2010; Yan et al. 2013). Key morphological features for cysts examined under a compound light microscope included color, underbridge in the vulval cone, semifenestrae shape, and development of bullae. The polymerase chain reaction (PCR) products of DNA extracted from cysts were digested with six restriction endonucleases (TaqI, HinfI, PstI, HaeIII, $R s a \mathrm{I}$, and $A l u \mathrm{I})$ and the species of Heterodera was determined by comparing the restriction pattern with those of nine pure Heterodera control species (H. avenae, $H$. filipjevi, $H$. latipons, and $H$. schachtii) in agarose gels by electrophoresis.

Statistical analyses. Grain yield and test weight over 2 years were analyzed using three-way analysis of variance (ANOVA) for individual experiments, with year as the main plot, cultivar as the subplot, nematicide treatment as the subsubplot, and replicates as blocks. ANOVA was performed using CoStat Statistical Software (Co-Stat v. 6.400; CoHort Software, Monterey, CA). Percent yield increase for each pair of control and nematicide treatments for individual cultivars within each experiment were analyzed using two-way ANOVA, with year as main plot, cultivar as the subplot, and replicates as blocks. Disease incidence and number of white females per plant and eggs per kilogram of soil after harvest only in the control (nonnematicide treatment) plots were also analyzed by two-way ANOVA. When treatment means were significant at $\alpha<0.05$, means were separated using the Tukey's honestly significant difference (HSD) test. Analyses were performed on nematode density data normalized by using the $\ln (x+1)$ transformation. Logarithmic means were back transformed into real numbers for presentation in the tables. Means of ordinate data for root knotting severity were analyzed by the Kruskal-Wallis Test. When the Pearson's $\chi^{2}$ value for the experiment was significant at $\alpha<0.05$, the treatments were examined pairwise to determine which treatments differed significantly. Six cultivars were sampled from both control and nematicide-treated plots each year, and those cultivars were analyzed separately to examine effects of year, cultivar, and nematicide treatments. Data for those six cultivars were analyzed using year as the main plot factor, cultivar as the subplot factor, and nematicide as the subsubplot factor. Because the main effect of year is highly significant in most analyses, the data for each of the three trials were also analyzed for individual years. Data are reported as the means and standard error of the means for trials performed during each year.

\section{Results}

Preplant nematode density. $H$. avenae was the only Heterodera sp. detected. The initial density of $H$. avenae across the three-trial block during 2013 was 22,176 eggs plus juveniles/kg of soil. During 2014 , the mean densities for the three experimental blocks were $3,516,27,000$, and 4,980 eggs plus juveniles/kg for two-row feed, two-row malt, and six-row barley, respectively.

Plant growth and development. Soil temperature at the depth of seed placement was $8.9^{\circ} \mathrm{C}$ in 2013 and $6.7^{\circ} \mathrm{C}$ in 2014 . The estimated dates of seedling emergence $\left(0^{\circ} \mathrm{C}\right.$ base for GDD) were 20 May 2013 and 10 May 2014, which were 22 and 20 days after planting, respectively. Accumulation of heat units during early spring (until 1 June) was greater during 2014 than during 2013. After 1 June, the GGD accumulation was more rapid during 2013 than during 2014. Initiation of anthesis was estimated to have occurred on 25 June 2013 and 1 July 2014.

Incidence and severity of root-knot symptom. Barley roots were sampled to determine incidence and severity of the root-knotting symptom and to count numbers of swollen white $H$. avenae females on 13 July 2013,59 days or 619 GDD $\left(8^{\circ} \mathrm{C}\right.$ base $)$ after seedling emergence and 18 days after anthesis. During 2014, the root sampling was performed on 23 July 2014,79 days or 633 GDD $\left(8^{\circ} \mathrm{C}\right.$ base) after seedling emergence and 23 days after anthesis. Disease incidence was near $100 \%$ for all plants in these experiments. The root-knotting symptom occurred on 98 to $100 \%$ of the plants of all cultivars in each experiment during both years, and in the nematicide-treated plots as well as in the control treatment (data not presented).

Severity of the root-knotting symptom in the control treatment of each experiment differed significantly $(P<0.0001)$ for the main effect of year but did not differ significantly among cultivars $(P=0.38)$, and the year-cultivar interaction was also not significant. For the two cultivars in which roots were also evaluated in the nematicide treatment, the effects of year and nematicide treatment were each significant $(P<0.0001)$ and the effect of cultivar was not significant $(P=$ 0.23 ). The mean severity of the root-knotting symptom in each experiment was greater during 2014 than during 2013 (Tables 1 to 3) and was greater in the control plots than in the nematicide-treated plots (data not shown). The control and nematicide treatments had mean severity ratings of 4.5 and $3.8\left(\mathrm{HSD}_{0.05}=0.4\right)$, respectively, for the two two-row feed barley cultivars; 4.6 and $4.3\left(\mathrm{HSD}_{0.05}=0.2\right)$, respectively, for the two two-row malt barley cultivars; and 4.5 and $2.9\left(\mathrm{HSD}_{0.05}=0.5\right)$, respectively, for the two six-row barley cultivars.

White females on roots. Numbers of newly produced $H$. avenae swollen white females were significantly $(P<0.01)$ influenced by the effect of year in each experiment and by the main effect of cultivar in only the two-row malting barley experiment. The year-cultivar 
interaction was significant for the malting barley experiment. Numbers of white females were greater during 2013 than during 2014 for the two-row malting barley (Table 2). For the two cultivars in which white females were quantified in the nematicide treatment, the main treatment effect for nematicide was the only significant treatment effect for the two-row feed barley experiment $(P<0.0001)$. In that experiment, none of the interactions were significant. In the two-row malt barley experiment, the treatment effects for year and nematicide were significant $(P<0.001)$, as was the year-nematicide interaction $(P<0.01)$. Likewise, the effects of year and nematicide were each significant $(P<0.05)$ for the six-row barley experiment. In each case, the numbers of white females were much higher on roots in the control plots than in the nematicide-treated plots. The mean numbers of white females in the control and nematicide treatments were 9.4 and $1.2\left(\mathrm{HSD}_{0.05}=4.1\right)$, respectively, for the two two-row feed barley cultivars; 24.8 and $1.4\left(\mathrm{HSD}_{0.05}=17.4\right)$, respectively, for the two two-row malt barley cultivars; and 16.3 and $1.9\left(\mathrm{HSD}_{0.05}=3.4\right)$, respectively, for the two six-row barley cultivars.

Numbers of swollen white females extracted and counted during 2014 were too low to provide reliable distinctions among cultivars.

Table 1. Evaluation of 16 two-row spring feed barley cultivars for resistance and tolerance to Heterodera avenae in two fields near St. Anthony, ID during 2013 and $2014^{\mathrm{s}}$

\begin{tabular}{|c|c|c|c|c|c|c|c|c|}
\hline \multirow[b]{2}{*}{ Cultivar } & \multirow[b]{2}{*}{ Severity $^{t}$} & \multirow[b]{2}{*}{ Females $^{\mathbf{u}}$} & \multirow[b]{2}{*}{ Eggs/kg of soilv } & \multicolumn{3}{|c|}{ Grain yield (kg/ha) } & \multirow[b]{2}{*}{$\operatorname{Res}^{\mathbf{y}}$} & \multirow[b]{2}{*}{ Tol $^{\mathbf{z}}$} \\
\hline & & & & Control & Treated $^{w}$ & Incr. $(\%)^{x}$ & & \\
\hline \multicolumn{9}{|l|}{2013} \\
\hline 08ID1549 & $4.0 \pm 0.0 \mathrm{a}$ & $11.30 \pm 3.5 \mathrm{a}$ & $\ldots$ & $4,232 \pm 467 \mathrm{a}-\mathrm{c}$ & $5,047 \pm 79 \mathrm{ab}$ & $23.5 \pm 13.0 \mathrm{a}$ & MS & MI \\
\hline 08ID2661 & $4.1 \pm 0.1 \mathrm{a}$ & $22.1 \pm 15.5 \mathrm{a}$ & $\ldots$ & $4,706 \pm 557 \mathrm{ab}$ & $5,349 \pm 831 \mathrm{ab}$ & $12.1 \pm 4.6 \mathrm{a}$ & S & MI \\
\hline Baronesse & $4.2 \pm 0.1 \mathrm{a}$ & $11.7 \pm 4.8 \mathrm{a}$ & $165,036 \pm 22,942 a$ & $4,991 \pm 490 \mathrm{ab}$ & $5,748 \pm 576 \mathrm{a}$ & $15.4 \pm 4.2 \mathrm{a}$ & MS & MI \\
\hline CDC Fibar & $4.0 \pm 0.1 \mathrm{a}$ & $7.9 \pm 1.5 \mathrm{a}$ & $\ldots$ & $3,730 \pm 318 b c$ & $3,875 \pm 180 \mathrm{bc}$ & $5.0 \pm 4.5 \mathrm{a}$ & MS & $\mathrm{T}$ \\
\hline CDC McGwire & $3.9 \pm 0.1 \mathrm{a}$ & $23.6 \pm 14.7 \mathrm{a}$ & $\ldots$ & $3,845 \pm 445 b c$ & $3,872 \pm 366 a-c$ & $12.1 \pm 4.0 \mathrm{a}$ & S & MT \\
\hline Champion & $3.8 \pm 0.2 \mathrm{a}$ & $10.9 \pm 4.0 \mathrm{a}$ & $223,305 \pm 54,378 a$ & $5,007 \pm 665 \mathrm{ab}$ & $5,375 \pm 607 \mathrm{ab}$ & $9.1 \pm 7.1 \mathrm{a}$ & MS & $\mathrm{T}$ \\
\hline Clearwater & $3.9 \pm 0.1 \mathrm{a}$ & $15.1 \pm 4.9 \mathrm{a}$ & $\ldots$ & $3,691 \pm 216 \mathrm{bc}$ & $4,394 \pm 310 \mathrm{a}-\mathrm{c}$ & $20.3 \pm 11.6 \mathrm{a}$ & S & MI \\
\hline Idagold II & $4.1 \pm 0.1 \mathrm{a}$ & $6.5 \pm 2.1 \mathrm{a}$ & $\ldots$ & $4,326 \pm 427 \mathrm{a}-\mathrm{c}$ & $4,399 \pm 445 \mathrm{a}-\mathrm{c}$ & $2.1 \pm 7.0 \mathrm{a}$ & MS & VT \\
\hline Julie & $4.2 \pm 0.1 \mathrm{a}$ & $12.3 \pm 1.4 \mathrm{a}$ & $\ldots$ & $4,646 \pm 239 \mathrm{ab}$ & $4,363 \pm 396 a-c$ & $-6.4 \pm 5.4 \mathrm{a}$ & S & VT \\
\hline Lenetah & $4.2 \pm 0.1 \mathrm{a}$ & $4.7 \pm 1.4 \mathrm{a}$ & $112,189 \pm 12,813 \mathrm{a}$ & $4,923 \pm 611 \mathrm{ab}$ & $5,101 \pm 695 \mathrm{ab}$ & $3.4 \pm 6.3 \mathrm{a}$ & MR & VT \\
\hline RWA 1758 & $4.3 \pm 0.1 \mathrm{a}$ & $9.5 \pm 4.6 \mathrm{a}$ & $\ldots$ & $5,303 \pm 834 \mathrm{a}$ & $5,471 \pm 446 \mathrm{a}$ & $8.7 \pm 11.6 \mathrm{a}$ & MS & $\mathrm{T}$ \\
\hline Spaulding & $4.0 \pm 0.3 \mathrm{a}$ & $43.6 \pm 21.6 \mathrm{a}$ & $113,717 \pm 6,538 \mathrm{a}$ & $4,668 \pm 335 a b$ & $5,329 \pm 97 a b$ & $14.3 \pm 3.2 \mathrm{a}$ & VS & MT \\
\hline Tetonia & $4.2 \pm 0.1 \mathrm{a}$ & $26.9 \pm 11.0 \mathrm{a}$ & $92,081 \pm 20,324 \mathrm{a}$ & $5,346 \pm 325 \mathrm{a}$ & $5,416 \pm 172 a$ & $2.0 \pm 3.7 \mathrm{a}$ & VS & VT \\
\hline Transit & $4.2 \pm 0.1 \mathrm{a}$ & $9.7 \pm 6.8 \mathrm{a}$ & $\ldots$ & $3,124 \pm 424 \mathrm{c}$ & $3,299 \pm 444 \mathrm{c}$ & $6.2 \pm 7.1 \mathrm{a}$ & MS & $\mathrm{T}$ \\
\hline Vespa & $4.2 \pm 0.1 \mathrm{a}$ & $21.1 \pm 6.2 \mathrm{a}$ & $\ldots$ & $5,522 \pm 332 \mathrm{a}$ & $5,626 \pm 451 \mathrm{a}$ & $1.9 \pm 5.7 \mathrm{a}$ & S & VT \\
\hline Xena & $4.2 \pm 0.1 \mathrm{a}$ & $7.6 \pm 3.2 \mathrm{a}$ & $\ldots$ & $5,527 \pm 368 \mathrm{a}$ & $5,546 \pm 403 a$ & $0.3 \pm 4.6 \mathrm{a}$ & MS & VT \\
\hline Mean & 4.1 & 15.3 & 117,721 & 4,600 & 4,912 & 8.1 & $S$ & $\mathrm{~T}$ \\
\hline$P>F$ & 0.5567 & 0.3021 & 0.4501 & $<0.0001$ & $<0.0001$ & 0.3033 & $\ldots$ & $\ldots$ \\
\hline $\mathrm{HSD}_{0.05}$ & ns & ns & ns & 1,409 & 845 & ns & $\ldots$ & $\ldots$ \\
\hline \multicolumn{9}{|l|}{2014} \\
\hline 08ID1549 & $4.1 \pm 0.2 \mathrm{a}$ & $2.6 \pm 0.4 \mathrm{a}$ & $309 \pm 188 \mathrm{a}$ & $4,025 \pm 284 \mathrm{c}-\mathrm{e}$ & $4,428 \pm 324 \mathrm{c}-\mathrm{f}$ & $8.7 \pm 4.1 \mathrm{a}$ & $\mathrm{R}$ & $\mathrm{T}$ \\
\hline 08ID2661 & $5.0 \pm 0.0 \mathrm{a}$ & $1.3 \pm 0.5 \mathrm{a}$ & $191 \pm 110 \mathrm{a}$ & $4,346 \pm 270 \mathrm{~b}-\mathrm{d}$ & $4,882 \pm 240$ b-f & $12.6 \pm 3.0 \mathrm{a}$ & $\mathrm{R}$ & MT \\
\hline Baronesse & $5.0 \pm 0.0 \mathrm{a}$ & $4.9 \pm 2.8 \mathrm{a}$ & $327 \pm 140 \mathrm{a}$ & $4,758 \pm 163 \mathrm{a}-\mathrm{c}$ & $4,851 \pm 150 \mathrm{~b}-\mathrm{f}$ & $2.1 \pm 1.9 \mathrm{a}$ & MR & VT \\
\hline CDC Fibar & $4.4 \pm 0.2 \mathrm{a}$ & $1.5 \pm 1.0 \mathrm{a}$ & $573 \pm 280 \mathrm{a}$ & $3,019 \pm 290 \mathrm{e}$ & $3,869 \pm 120 \mathrm{fg}$ & $31.5 \pm 14.2 \mathrm{a}$ & $\mathrm{R}$ & I \\
\hline CDC McGwire & $5.0 \pm 0.0 \mathrm{a}$ & $1.5 \pm 0.6 \mathrm{a}$ & $264 \pm 78 \mathrm{a}$ & $4,138 \pm 487 b-e$ & $4,401 \pm 333 \mathrm{~d}-\mathrm{f}$ & $11.6 \pm 13.4 \mathrm{a}$ & $\mathrm{R}$ & MT \\
\hline Champion & $5.0 \pm 0.0 \mathrm{a}$ & $3.2 \pm 1.7 \mathrm{a}$ & $427 \pm 283 \mathrm{a}$ & $4,620 \pm 162 a-c$ & $4,854 \pm 190$ b-f & $5.3 \pm 5.4 \mathrm{a}$ & MR & $\mathrm{T}$ \\
\hline Clearwater & $5.0 \pm 0.0 \mathrm{a}$ & $1.3 \pm 0.6 \mathrm{a}$ & $491 \pm 177 \mathrm{a}$ & $3,226 \pm 317 \mathrm{de}$ & $3,968 \pm 235 \mathrm{e}-\mathrm{g}$ & $25.9 \pm 12.2 \mathrm{a}$ & $\mathrm{R}$ & MI \\
\hline Idagold II & $5.0 \pm 0.0 \mathrm{a}$ & $3.9 \pm 1.9 \mathrm{a}$ & $339 \pm 231 \mathrm{a}$ & $4,825 \pm 272 a-c$ & $5,729 \pm 108 \mathrm{ab}$ & $19.1 \pm 4.2 \mathrm{a}$ & MR & MI \\
\hline Julie & $5.0 \pm 0.0 \mathrm{a}$ & $0.3 \pm 0.2 \mathrm{a}$ & $267 \pm 165 \mathrm{a}$ & $4,048 \pm 148 \mathrm{c}-\mathrm{e}$ & $4,426 \pm 158 c-f$ & $9.4 \pm 4.6 \mathrm{a}$ & VR & $\mathrm{T}$ \\
\hline Lenetah & $4.7 \pm 0.1 \mathrm{a}$ & $0.9 \pm 0.1 \mathrm{a}$ & $145 \pm 64 \mathrm{a}$ & $4,429 \pm 260 a-d$ & $5,115 \pm 225 \mathrm{a}-\mathrm{d}$ & $16.2 \pm 7.7 \mathrm{a}$ & VR & MI \\
\hline RWA 1758 & $5.0 \pm 0.0 \mathrm{a}$ & $6.3 \pm 3.1 \mathrm{a}$ & $345 \pm 48 a$ & $4,880 \pm 99 a-c$ & $4,912 \pm 122 b-\mathrm{e}$ & $0.7 \pm 2.7 \mathrm{a}$ & MS & VT \\
\hline Spaulding & $4.3 \pm 0.3 \mathrm{a}$ & $1.7 \pm 0.8 \mathrm{a}$ & $200 \pm 92 \mathrm{a}$ & $4,978 \pm 253 \mathrm{a}-\mathrm{c}$ & $5,451 \pm 185 \mathrm{a}-\mathrm{c}$ & $9.9 \pm 3.2 \mathrm{a}$ & $\mathrm{R}$ & $\mathrm{T}$ \\
\hline Tetonia & $4.1 \pm 0.4 \mathrm{a}$ & $3.1 \pm 1.2 \mathrm{a}$ & $185 \pm 27 \mathrm{a}$ & $5,001 \pm 219 a-c$ & $5,335 \pm 140 \mathrm{a}-\mathrm{d}$ & $7.2 \pm 3.4 \mathrm{a}$ & MR & $\mathrm{T}$ \\
\hline Transit & $5.0 \pm 0.0 \mathrm{a}$ & $3.1 \pm 1.2 \mathrm{a}$ & $385 \pm 106 \mathrm{a}$ & $2,666 \pm 169 \mathrm{e}$ & $3,289 \pm 141 \mathrm{~g}$ & $14.8 \pm 1.4 \mathrm{a}$ & MR & MT \\
\hline Vespa & $4.9 \pm 0.1 \mathrm{a}$ & $0.6 \pm 0.2 \mathrm{a}$ & $373 \pm 146 a$ & $5,650 \pm 155 \mathrm{a}$ & $5,869 \pm 99 \mathrm{ab}$ & $4.1 \pm 2.1 \mathrm{a}$ & VR & VT \\
\hline Xena & $5.0 \pm 0.0 \mathrm{a}$ & $0.5 \pm 0.3 \mathrm{a}$ & $130 \pm 99 \mathrm{a}$ & $5,406 \pm 282 a b$ & $5,965 \pm 350 \mathrm{a}$ & $10.4 \pm 6.8 \mathrm{a}$ & VR & MT \\
\hline Mean & 4.8 & 2.3 & 309 & 4,391 & 4,834 & 11.9 & $\mathrm{R}$ & MT \\
\hline$P>F$ & 0.4488 & 0.0585 & 0.7817 & $<0.0001$ & $<0.0001$ & 0.2230 & $\ldots$ & $\ldots$ \\
\hline $\mathrm{HSD}_{0.05}$ & ns & ns & ns & 707 & 578 & ns & $\ldots$ & $\ldots$ \\
\hline
\end{tabular}

$\mathrm{s}$ Means and standard error of the mean for three replicates of each disease parameter and four replicates of grain parameters. Means followed by the same letter within a column did not differ significantly at $\alpha=0.05$ as determined by Tukey's honestly significant difference (HSD) test; ns $=$ not significant. Means of severity data for the root knot symptom were analyzed by the Kruskal-Wallis test and, when the Pearson's $\chi^{2}$ value for the experiment was significant at $\alpha<0.05$, cultivars were examined pairwise to determine which treatments differed significantly.

${ }^{\mathrm{t}}$ Root knot severity ratings for control (no-nematicide) treatment only, with a scale of $1=$ no evidence of damage, $2=1$ to $3 \mathrm{knots} / \mathrm{root}$ system, $3=3$ to $5 \mathrm{knots}, 4=$ $>5$ knots and $<20 \%$ reduction in plant height or root mass, and $5=>5$ knots and $>20 \%$ reduction in plant height or root mass. More than $98 \%$ of all cultivars exhibited the root knotting symptom on at least one root (e.g., the incidence was 98 to $100 \%$ ).

" Number of $H$. avenae white females produced per plant for the control (no-nematicide) treatment only; sampling was performed at about the time of anthesis.

$\checkmark$ Number of $H$. avenae eggs per kilogram of soil for the control (no-nematicide) treatment only; extraction of cysts was performed from soil that was dry following harvest.

w Nematicide treatment included application of aldicarb (aldicarb at $4.2 \mathrm{~kg} / \mathrm{ha}$ ) in the seed row at the time of planting.

$x$ Percent increase in grain yield due to application of nematicide.

y Phenotypic resistance reaction: very resistant (VR; $\leq 1$ swollen female/plant), resistant (R; 1.1 to 3 ), moderately resistant (MR; 3.1 to 6), moderately susceptible (MS; 6.1 to 12 ), susceptible ( $; 12.1$ to 25 ), or very susceptible (VS; $>25$ ).

${ }^{z}$ Phenotypic tolerance reaction: very tolerant (VT; $<5 \%$ yield response to nematicide), tolerant (T; 5 to $10 \%$ ), moderately tolerant (MT; 10 to $15 \%$ ), moderately intolerant (MI; 15 to $30 \%$ ), intolerant (I; 30 to $50 \%$ ), or very intolerant (VI; >50\%). 
During 2013, 'Lenetah' was the only two-row feed barley cultivar to be rated as moderately resistant to $H$. avenae, and no cultivars were rated as resistant or very resistant (Table 1). 'Odyssey' was the only two-row malt barley to be rated as resistant during 2013 (Table 2).
Numbers of white females in the six-row barley experiment were low each year and there was no consensus of resistance and susceptibility ratings between years (Table 3 ). For instance, the five six-row barley cultivars that were rated as resistant or moderately resistant

Table 2. Evaluation of 19 two-row malt barley cultivars for resistance and tolerance to Heterodera avenae in two fields near St. Anthony, ID during 2013 and $2014^{\mathrm{s}}$

\begin{tabular}{|c|c|c|c|c|c|c|c|c|}
\hline \multirow[b]{2}{*}{ Cultivar } & \multirow[b]{2}{*}{ Severity $^{t}$} & \multirow[b]{2}{*}{ Females $^{\mathbf{u}}$} & \multirow[b]{2}{*}{ Eggs/kg of soilv } & \multicolumn{3}{|c|}{ Grain yield (kg/ha) } & \multirow[b]{2}{*}{$\operatorname{Res}^{\mathbf{y}}$} & \multirow[b]{2}{*}{ Tol $^{\mathbf{Z}}$} \\
\hline & & & & Control & Treated $^{w}$ & Incr. $(\%)^{\mathrm{x}}$ & & \\
\hline \multicolumn{9}{|l|}{2013} \\
\hline 2Ab04-X001084-27 & $4.1 \pm 0.1 \mathrm{a}$ & $45.5 \pm 12.8 \mathrm{a}$ & $\ldots$ & $4,834 \pm 250 \mathrm{ab}$ & $5,067 \pm 311 \mathrm{a}$ & $5.8 \pm 9.0 \mathrm{a}$ & VS & $\mathrm{T}$ \\
\hline 2Ab07-X031098-31 & $4.0 \pm 0.1 \mathrm{a}$ & $31.0 \pm 16.6 \mathrm{ab}$ & $\ldots$ & $4,712 \pm 573 a-c$ & $4,938 \pm 397 \mathrm{a}$ & $6.3 \pm 5.0 \mathrm{a}$ & VS & $\mathrm{T}$ \\
\hline 2B05-0811 (B0811) & $4.2 \pm 0.1 \mathrm{a}$ & $20.1 \pm 6.9 \mathrm{ab}$ & $\ldots$ & $4,172 \pm 612$ a-f & $3,784 \pm 539 b-d$ & $-7.4 \pm 11.1 \mathrm{a}$ & S & VT \\
\hline ABI Voyager & $4.3 \pm 0.0 \mathrm{a}$ & $92.7 \pm 43.2 \mathrm{a}$ & $210,432 \pm 22,374 \mathrm{a}$ & $3,507 \pm 651$ ef & $4,478 \pm 854 a-d$ & $28.0 \pm 8.3 \mathrm{a}$ & VS & MI \\
\hline B1202 & $4.0 \pm 0.0 \mathrm{a}$ & $55.6 \pm 23.8 \mathrm{a}$ & $\ldots$ & $4,094 \pm 592 \mathrm{a}-\mathrm{f}$ & $4,442 \pm 660 \mathrm{a}-\mathrm{d}$ & $8.5 \pm 3.6 \mathrm{a}$ & VS & $\mathrm{T}$ \\
\hline Conrad & $4.1 \pm 0.1 \mathrm{a}$ & $19.8 \pm 6.1 \mathrm{ab}$ & $139,642 \pm 15,425 \mathrm{a}$ & $3,961 \pm 756 b-f$ & $4,527 \pm 939 a-c$ & $13.5 \pm 9.6 \mathrm{a}$ & S & MT \\
\hline Copeland & $4.2 \pm 0.1 \mathrm{a}$ & $37.6 \pm 9.8 \mathrm{a}$ & $\ldots$ & $5,058 \pm 514 a$ & $5,140 \pm 569 a$ & $1.7 \pm 5.6 \mathrm{a}$ & VS & VT \\
\hline Genie & $4.2 \pm 0.0 \mathrm{a}$ & $66.7 \pm 5.0 \mathrm{a}$ & $\ldots$ & $4,283 \pm 515$ a-e & $4,893 \pm 480 a$ & $15.0 \pm 3.5 \mathrm{a}$ & VS & MT \\
\hline Harrington & $4.2 \pm 0.1 \mathrm{a}$ & $40.5 \pm 6.3 \mathrm{a}$ & $\ldots$ & $3,278 \pm 492 \mathrm{f}$ & $3,522 \pm 367 \mathrm{~d}$ & $10.1 \pm 7.5 \mathrm{a}$ & VS & MT \\
\hline Hockett & $4.4 \pm 0.0 \mathrm{a}$ & $51.7 \pm 14.5 \mathrm{a}$ & $\ldots$ & $4,263 \pm 432$ a-f & $4,718 \pm 426 \mathrm{ab}$ & $11.9 \pm 8.4 \mathrm{a}$ & VS & MT \\
\hline LCS 1820 & $4.2 \pm 0.1 \mathrm{a}$ & $18.8 \pm 7.3 \mathrm{ab}$ & $231,212 \pm 32,425 \mathrm{a}$ & $3,723 \pm 619 c-f$ & $3,829 \pm 630 b-d$ & $2.9 \pm 0.5 \mathrm{a}$ & S & VT \\
\hline Meredith & $4.2 \pm 0.0 \mathrm{a}$ & $25.3 \pm 5.1 \mathrm{a}$ & $\ldots$ & $3,964 \pm 742$ b-f & $4,619 \pm 561 \mathrm{a}-\mathrm{c}$ & $25.0 \pm 17.6 \mathrm{a}$ & VS & MI \\
\hline Merem & $4.1 \pm 0.1 \mathrm{a}$ & $26.8 \pm 12.4 \mathrm{ab}$ & $\ldots$ & $4,631 \pm 426 \mathrm{a}-\mathrm{c}$ & $4,657 \pm 365 a b$ & $1.1 \pm 3.0 \mathrm{a}$ & VS & VT \\
\hline Merit & $4.1 \pm 0.1 \mathrm{a}$ & $27.9 \pm 11.5 \mathrm{a}$ & $\ldots$ & $3,570 \pm 466 \mathrm{~d}-\mathrm{f}$ & $3,868 \pm 580 \mathrm{~b}-\mathrm{d}$ & $7.4 \pm 5.3 \mathrm{a}$ & VS & $\mathrm{T}$ \\
\hline Merit 57 & $4.1 \pm 0.1 \mathrm{a}$ & $67.9 \pm 37.3 \mathrm{a}$ & $163,622 \pm 5,278 \mathrm{a}$ & $3,388 \pm 881$ ef & $3,658 \pm 937 \mathrm{~cd}$ & $8.0 \pm 4.3 \mathrm{a}$ & VS & $\mathrm{T}$ \\
\hline Metcalf & $4.1 \pm 0.1 \mathrm{a}$ & $48.9 \pm 32.7 \mathrm{a}$ & $232,572 \pm 37,765 \mathrm{a}$ & $3,861 \pm 665 b-f$ & $4,572 \pm 869 a-c$ & $17.8 \pm 6.7 \mathrm{a}$ & VS & MI \\
\hline Odyssey & $4.1 \pm 0.1 \mathrm{a}$ & $1.8 \pm 0.4 \mathrm{~b}$ & $24,593 \pm 6,560 \mathrm{~b}$ & $4,511 \pm 682 a-d$ & $5,354 \pm 951 a$ & $17.3 \pm 10.3 \mathrm{a}$ & $\mathrm{R}$ & MI \\
\hline Overture & $4.2 \pm 0.0 \mathrm{a}$ & $33.4 \pm 3.8 \mathrm{a}$ & $\ldots$ & $4,237 \pm 969$ a-f & $4,404 \pm 536$ a-d & $4.5 \pm 3.9 \mathrm{a}$ & VS & VT \\
\hline Pinnacle & $4.1 \pm 0.1 \mathrm{a}$ & $37.1 \pm 21.4 \mathrm{a}$ & $\ldots$ & $3,562 \pm 814 d-f$ & $3,732 \pm 808 \mathrm{~b}-\mathrm{d}$ & $6.4 \pm 3.4 \mathrm{a}$ & VS & $\mathrm{T}$ \\
\hline Mean & 4.2 & 39.5 & 167,012 & 4,085 & 4,432 & 9.7 & VS & $\mathrm{T}$ \\
\hline$P>F$ & 0.2930 & 0.0018 & 0.0004 & 0.0180 & 0.0058 & 0.2651 & $\ldots$ & $\ldots$ \\
\hline $\mathrm{HSD}_{0.05}$ & $\mathrm{~ns}$ & $\ldots$ & $\ldots$ & 997 & 996 & $\mathrm{Ns}$ & $\cdots$ & $\cdots$ \\
\hline \multicolumn{9}{|l|}{2014} \\
\hline 2Ab04-X001084-27 & $4.7 \pm 0.3 \mathrm{a}$ & $1.9 \pm 0.8 \mathrm{a}$ & $364 \pm 229 a$ & $4,876 \pm 366 \mathrm{a}-\mathrm{d}$ & $4,844 \pm 221 \mathrm{c}$ & $0.2 \pm 4.4 b$ & $\mathrm{R}$ & VT \\
\hline 2Ab07-X031098-31 & $4.9 \pm 0.1 \mathrm{a}$ & $3.1 \pm 1.0 \mathrm{a}$ & $282 \pm 118 \mathrm{a}$ & $4,816 \pm 125 \mathrm{a}-\mathrm{d}$ & $5,060 \pm 99 a-c$ & $5.1 \pm 1.3 b$ & MR & $\mathrm{T}$ \\
\hline 2B05-0811 (B0811) & $4.7 \pm 0.3 \mathrm{a}$ & $6.7 \pm 1.9 \mathrm{a}$ & $709 \pm 481 \mathrm{a}$ & $4,884 \pm 291 \mathrm{a}-\mathrm{d}$ & $5,370 \pm 275 a-c$ & $10.3 \pm 3.6 b$ & MS & MT \\
\hline ABI Voyager & $5.0 \pm 0.0 \mathrm{a}$ & $2.1 \pm 1.4 \mathrm{a}$ & $182 \pm 66 \mathrm{a}$ & $4,594 \pm 343 a-d$ & $4,998 \pm 291 b c$ & $10.0 \pm 7.4 \mathrm{~b}$ & $\mathrm{R}$ & $\mathrm{T}$ \\
\hline B1202 & $5.0 \pm 0.0 \mathrm{a}$ & $1.5 \pm 0.8 \mathrm{a}$ & $642 \pm 270 \mathrm{a}$ & $3,747 \pm 136 \mathrm{~d}$ & $5,641 \pm 301 \mathrm{a}-\mathrm{c}$ & $51.9 \pm 13.2 \mathrm{a}$ & $\mathrm{R}$ & VI \\
\hline Conrad & $5.0 \pm 0.0 \mathrm{a}$ & $2.2 \pm 1.4 \mathrm{a}$ & $273 \pm 192 \mathrm{a}$ & $4,352 \pm 324 \mathrm{a}-\mathrm{d}$ & $5,125 \pm 227 \mathrm{a}-\mathrm{c}$ & $20.0 \pm 11.4 \mathrm{ab}$ & $\mathrm{R}$ & MI \\
\hline Copeland & $4.9 \pm 0.1 \mathrm{a}$ & $10.1 \pm 8.8 \mathrm{a}$ & $364 \pm 271 a$ & $4,438 \pm 250 a-d$ & $4,724 \pm 203 \mathrm{c}$ & $7.2 \pm 6.2 \mathrm{~b}$ & MS & $\mathrm{T}$ \\
\hline Genie & $5.0 \pm 0.0 \mathrm{a}$ & $1.1 \pm 0.7 \mathrm{a}$ & $185 \pm 128 \mathrm{a}$ & $5,026 \pm 188 \mathrm{a}-\mathrm{d}$ & $5,334 \pm 289 a-c$ & $6.3 \pm 5.8 b$ & $\mathrm{R}$ & $\mathrm{T}$ \\
\hline Harrington & $4.7 \pm 0.1 \mathrm{a}$ & $2.6 \pm 1.2 \mathrm{a}$ & $736 \pm 129 a$ & $4,176 \pm 61 b-d$ & $4,609 \pm 177 \mathrm{c}$ & $10.5 \pm 5.3 b$ & $\mathrm{R}$ & MT \\
\hline Hockett & $5.0 \pm 0.0 \mathrm{a}$ & $2.1 \pm 1.4 \mathrm{a}$ & $400 \pm 87 \mathrm{a}$ & $4,012 \pm 220 \mathrm{~cd}$ & $4,840 \pm 176 \mathrm{c}$ & $21.2 \pm 4.9 \mathrm{ab}$ & $\mathrm{R}$ & MI \\
\hline LCS1820 & $4.5 \pm 0.1 \mathrm{a}$ & $0.8 \pm 0.3 \mathrm{a}$ & $91 \pm 66 \mathrm{a}$ & $4,850 \pm 285 a-d$ & $5,534 \pm 453 a-c$ & $14.8 \pm 10.0 \mathrm{~b}$ & VR & MT \\
\hline Meredith & $4.9 \pm 0.1 \mathrm{a}$ & $3.4 \pm 1.0 \mathrm{a}$ & $527 \pm 224 \mathrm{a}$ & $4,492 \pm 252 \mathrm{a}-\mathrm{d}$ & $4,559 \pm 90 \mathrm{c}$ & $2.1 \pm 3.9 \mathrm{~b}$ & MR & VT \\
\hline Merem & $5.0 \pm 0.0 \mathrm{a}$ & $4.6 \pm 2.1 \mathrm{a}$ & $424 \pm 69 a$ & $4,199 \pm 319 a-d$ & $4,542 \pm 238 \mathrm{c}$ & $10.1 \pm 8.8 b$ & MR & MT \\
\hline Merit & $5.0 \pm 0.0 \mathrm{a}$ & $7.1 \pm 1.8 \mathrm{a}$ & $948 \pm 658 a$ & $5,093 \pm 408 \mathrm{a}-\mathrm{c}$ & $5,064 \pm 240 a-c$ & $0.2 \pm 3.3 \mathrm{~b}$ & MS & VT \\
\hline Merit 57 & $4.7 \pm 0.3 \mathrm{a}$ & $5.1 \pm 2.5 \mathrm{a}$ & $448 \pm 390 a$ & $5,211 \pm 244 a-c$ & $5,447 \pm 160 a-c$ & $5.1 \pm 4.5 \mathrm{~b}$ & MR & $\mathrm{T}$ \\
\hline Metcalf & $5.0 \pm 0.0 \mathrm{a}$ & $1.7 \pm 1.0 \mathrm{a}$ & $284 \pm 88 \mathrm{a}$ & $4,143 \pm 198 b-d$ & $4,790 \pm 257 \mathrm{c}$ & $15.8 \pm 4.5 b$ & $\mathrm{R}$ & MI \\
\hline Odyssey & $5.0 \pm 0.0 \mathrm{a}$ & $0.1 \pm 0.1 \mathrm{a}$ & $197 \pm 148 a$ & $5,527 \pm 236 a$ & $6,264 \pm 184 \mathrm{a}$ & $13.8 \pm 4.6 \mathrm{~b}$ & VR & MT \\
\hline Overture & $5.0 \pm 0.0 \mathrm{a}$ & $1.4 \pm 0.7 \mathrm{a}$ & $115 \pm 27 \mathrm{a}$ & $5,411 \pm 130 a b$ & $6,134 \pm 248 a b$ & $13.6 \pm 5.4 b$ & $\mathrm{R}$ & MT \\
\hline Pinnacle & $5.0 \pm 0.0 \mathrm{a}$ & $3.3 \pm 1.7 \mathrm{a}$ & $100 \pm 5 a$ & $4,443 \pm 314 a-d$ & $4,721 \pm 171 \mathrm{c}$ & $7.4 \pm 6.1 \mathrm{~b}$ & MR & $\mathrm{T}$ \\
\hline Mean & 4.9 & 3.2 & 382 & 4,647 & 5,137 & 11.9 & MR & MT \\
\hline$P>F$ & 0.2716 & 0.1036 & 0.2724 & 0.0001 & $<0.0001$ & 0.0011 & $\ldots$ & $\cdots$ \\
\hline $\mathrm{HSD}_{0.05}$ & $\mathrm{~ns}$ & Ns & ns & 730 & 670 & 18.8 & $\cdots$ & $\cdots$ \\
\hline
\end{tabular}

${ }^{\mathrm{s}}$ Means and standard error of the mean for three replicates of each disease parameter and four replicates of grain parameters. Means followed by the same letter within a column did not differ significantly at $\alpha=0.05$ as determined by Tukey's honestly significant difference (HSD) test; ns $=$ not significant. Means of severity data for the root knot symptom were analyzed by the Kruskal-Wallis test and, when the Pearson's $\chi^{2}$ value for the experiment was significant at $\alpha$ $<0.05$, cultivars were examined pairwise to determine which treatments differed significantly.

${ }^{\mathrm{t}}$ Root knot severity ratings for control (no-nematicide) treatment only, with a scale of $1=$ no evidence of damage, $2=1$ to $3 \mathrm{knots} /$ root system, $3=3$ to $5 \mathrm{knots}, 4=$ $>5$ knots and $<20 \%$ reduction in plant height or root mass, and $5=>5$ knots and $>20 \%$ reduction in plant height or root mass. More than $98 \%$ of all cultivars exhibited the root knotting symptom on at least one root (e.g., the incidence was 98 to $100 \%$ ).

u Number of $H$. avenae white females produced per plant for the control (no-nematicide) treatment only; sampling was performed at about the time of anthesis.

v Number of $H$. avenae eggs per kilogram of soil for the control (no-nematicide) treatment only; extraction of cysts was performed from soil that was dry following harvest.

w Nematicide treatment included application of aldicarb (aldicarb at $4.2 \mathrm{~kg} / \mathrm{ha}$ ) in the seed row at the time of planting.

$\mathrm{x}$ Percent increase in grain yield due to application of nematicide.

y Phenotypic resistance reaction: very resistant (VR; $\leq 1$ swollen female/plant), resistant (R; 1.1 to 3 ), moderately resistant (MR; 3.1 to 6$)$, moderately susceptible (MS; 6.1 to 12 ), susceptible (S; 12.1 to 25 ), or very susceptible (VS; $>25$ ).

${ }^{\mathrm{z}}$ Phenotypic tolerance reaction: very tolerant (VT; <5\% yield response to nematicide), tolerant (T; 5 to $10 \%$ ), moderately tolerant (MT; 10 to $15 \%$ ), moderately intolerant (MI; 15 to $30 \%$ ), intolerant (I; 30 to $50 \%$ ), or very intolerant (VI; $>50 \%$ ). 
during 2013 were each rated as moderately susceptible or susceptible during 2014. In contrast, the two cultivars that were rated as moderately resistant during 2014 were rated as moderately susceptible during 2013.

Postharvest density of $\boldsymbol{H}$. avenae eggs from cysts. Soil samples during 2013 were evaluated for five cultivars in the two-row feed barley experiment: 'Baronesse', 'Champion', Lenetah, 'Spaulding', and 'Tetonia'. There was a significant effect of nematicide treatment $(P<$ 0.0001 ) on mean egg density, with 141,265 and 64,519 eggs $/ \mathrm{kg}$ of soil in the control and nematicide treatments, respectively, which equated to a $54 \%$ reduction in $H$. avenae density in aldicarbtreated versus control plots (data not shown). The main effect for cultivar was not significant. A similar relationship occurred for the six cultivars examined in the two-row malting barley experiment $(167,002$ versus 76,894 eggs $/ \mathrm{kg} ; 54 \%$ reduction) and in the sixrow barley experiment $(58,212$ versus $12,600 \mathrm{eggs} / \mathrm{kg}$; $78 \%$ reduction). Cultivars examined in the two-row malting experiment included 'ABI Voyager', 'Conrad', 'LCS1820', 'Merit 57', 'Metcalf', and Odyssey. Cultivars of six-row barley tested included 'Celebration', 'Goldeneye', 'Legacy', 'Millenium', 'Morex', and 'Steptoe'.
Because the nematicide clearly reduced the density of $H$. avenae eggs in soil, with many or most of the remaining eggs in nematicide treatments representing eggs from cysts developed during earlier years, it was determined that sampling emphasis during 2014 should be focused on differences among cultivars in the control treatment. All cultivars in the control plots and two cultivars in the nematicidetreated plots were sampled during 2014. The cultivars sampled in the nematicide treatment were the same as those for which the root disease symptoms and numbers of swollen white females had been examined during 2013. The main effect of year was highly significant $(P<$ $0.0001)$ for all three experiments. The main effect for nematicide was also significant $(P<0.01)$ and the effect of cultivar was not significant in each experiment. None of the interactions were significant. When all cultivars in the control treatment were examined during 2014, the cultivar effect was not significant for any of the three experiments. The range of low to high $H$. avenae density for the two-row feed barley was from $56 \mathrm{eggs} / \mathrm{kg}$ of soil for 'Xena' to 209 eggs/kg for '08ID1549' $(P=0.78)$. The range for the two-row malt barley was from 100 eggs $/ \mathrm{kg}$ for 'Pinnacle' to $412 \mathrm{eggs} / \mathrm{kg}$ for 'Merem' $(P=0.27)$, and the range for the six-row barley was from $750 \mathrm{eggs} / \mathrm{kg}$ for 'Tradition' to $627 \mathrm{eggs} / \mathrm{kg}$ for '01 Ab9663' $(P=0.13)$

Table 3. Evaluation of four six-row feed barley and six six-row malting barley (M) cultivars for resistance and tolerance to Heterodera avenae in two fields near St. Anthony, ID during 2013 and $2014^{\mathrm{s}}$

\begin{tabular}{|c|c|c|c|c|c|c|c|c|}
\hline \multirow[b]{2}{*}{ Cultivar } & \multirow[b]{2}{*}{ Severity $^{t}$} & \multirow[b]{2}{*}{ Females ${ }^{u}$} & \multirow[b]{2}{*}{ Eggs $/ \mathrm{kg}$ of soil ${ }^{v}$} & \multicolumn{3}{|c|}{ Grain yield (kg/ha) } & \multirow[b]{2}{*}{$\operatorname{Res}^{\mathbf{y}}$} & \multirow[b]{2}{*}{ Tol $^{\mathrm{z}}$} \\
\hline & & & & Control & Treated $^{\mathrm{w}}$ & Incr. $(\%)^{x}$ & & \\
\hline \multicolumn{9}{|l|}{2013} \\
\hline Goldeneye & $4.2 \pm 0.0 \mathrm{a}$ & $7.9 \pm 4.7 \mathrm{a}$ & $35,563 \pm 1,702 \mathrm{a}$ & $6,238 \pm 597 a$ & $6,240 \pm 482 \mathrm{a}$ & $0.6 \pm 2.2 \mathrm{a}$ & MS & VT/MS \\
\hline Herald & $4.0 \pm 0.0 \mathrm{a}$ & $5.3 \pm 3.4 \mathrm{a}$ & $\ldots$ & $5,827 \pm 516 \mathrm{a}$ & $5,887 \pm 213 \mathrm{a}$ & $2.9 \pm 7.6 \mathrm{a}$ & MR & VT/MR \\
\hline Millennium & $4.2 \pm 0.0 \mathrm{a}$ & $2.2 \pm 0.5 \mathrm{a}$ & $81,816 \pm 8,814 a$ & $6,065 \pm 376 a$ & $6,026 \pm 214 a$ & $-0.3 \pm 4.9 \mathrm{a}$ & $\mathrm{R}$ & VT/R \\
\hline Steptoe & $4.2 \pm 0.3 \mathrm{a}$ & $2.9 \pm 1.4 \mathrm{a}$ & $58,233 \pm 1,717 \mathrm{a}$ & $4,855 \pm 297 \mathrm{a}$ & $5,728 \pm 741 \mathrm{a}$ & $17.8 \pm 11.6 \mathrm{a}$ & $\mathrm{R}$ & $\mathrm{MI} / \mathrm{R}$ \\
\hline $01 \mathrm{AB} 9663(\mathrm{M})$ & $3.9 \pm 0.1 \mathrm{a}$ & $8.8 \pm 8.3 \mathrm{a}$ & $\ldots$ & $6,447 \pm 506 a$ & $6,266 \pm 411 \mathrm{a}$ & $-2.4 \pm 3.0 \mathrm{a}$ & MS & VT/MS \\
\hline Celebration (M) & $3.9 \pm 0.1 \mathrm{a}$ & $9.3 \pm 6.8 \mathrm{a}$ & $63,734 \pm 6,104 \mathrm{a}$ & $4,910 \pm 515 a$ & $5,520 \pm 478 a$ & $14.2 \pm 10.1 \mathrm{a}$ & MS & MT/MS \\
\hline Legacy $(\mathrm{M})$ & $3.9 \pm 0.2 \mathrm{a}$ & $2.5 \pm 0.5 \mathrm{a}$ & $49,997 \pm 13,571 \mathrm{a}$ & $5,558 \pm 496 a$ & $6,080 \pm 91 \mathrm{a}$ & $11.8 \pm 9.4 \mathrm{a}$ & $\mathrm{R}$ & $\mathrm{MT} / \mathrm{R}$ \\
\hline Morex (M) & $4.2 \pm 0.1 \mathrm{a}$ & $13.1 \pm 6.8 \mathrm{a}$ & $59,929 \pm 2,005 \mathrm{a}$ & $5,280 \pm 257 \mathrm{a}$ & $5,293 \pm 342 \mathrm{a}$ & $0.0 \pm 1.6 \mathrm{a}$ & $S$ & VT/S \\
\hline Quest (M) & $3.8 \pm 0.3 \mathrm{a}$ & $8.2 \pm 1.6 \mathrm{a}$ & $\ldots$ & $5,413 \pm 535 \mathrm{a}$ & $5,190 \pm 383 a$ & $-3.3 \pm 3.4 \mathrm{a}$ & MS & VT/MS \\
\hline Tradition (M) & $3.9 \pm 0.2 \mathrm{a}$ & $5.7 \pm 2.3 \mathrm{a}$ & $\ldots$ & $5,032 \pm 396 a$ & $5,310 \pm 345 \mathrm{a}$ & $6.7 \pm 7.4 \mathrm{a}$ & MR & $\mathrm{T} / \mathrm{MR}$ \\
\hline Mean & 4.0 & 6.6 & 58,212 & 5,563 & 5,754 & 4.8 & MS & VT/MS \\
\hline$P>F$ & 0.5429 & 0.7669 & 0.5214 & 0.1948 & 0.4484 & 0.4128 & $\ldots$ & $\ldots$ \\
\hline $\mathrm{HSD}_{0.05}$ & ns & ns & $\mathrm{ns}$ & ns & ns & ns & $\ldots$ & $\ldots$ \\
\hline \multicolumn{9}{|l|}{2014} \\
\hline Goldeneye & $4.7 \pm 0.3 \mathrm{a}$ & $5.9 \pm 2.1 \mathrm{a}$ & $591 \pm 87 \mathrm{a}$ & $3,397 \pm 253 \mathrm{a}$ & $4,312 \pm 443 \mathrm{a}$ & $27.0 \pm 9.8 \mathrm{a}$ & MR & MI \\
\hline Herald & $5.0 \pm 0.0 \mathrm{a}$ & $17.4 \pm 0.8 \mathrm{a}$ & $457 \pm 236 \mathrm{a}$ & $3,844 \pm 471 \mathrm{a}$ & $4,067 \pm 319 a b$ & $8.3 \pm 9.1 \mathrm{a}$ & $S$ & $\mathrm{~T}$ \\
\hline Millennium & $4.7 \pm 0.3 \mathrm{a}$ & $10.1 \pm 5.1 \mathrm{a}$ & $221 \pm 53 \mathrm{a}$ & $4,139 \pm 338 a$ & $4,326 \pm 192 \mathrm{a}$ & $5.5 \pm 4.0 \mathrm{a}$ & MS & $\mathrm{T}$ \\
\hline Steptoe & $4.9 \pm 0.1 \mathrm{a}$ & $8.4 \pm 1.7 \mathrm{a}$ & $1,136 \pm 237 a$ & $3,817 \pm 280 a$ & $4,341 \pm 338 \mathrm{a}$ & $16.7 \pm 15.0 \mathrm{a}$ & MS & MI \\
\hline $01 \mathrm{AB} 9663$ (M) & $4.1 \pm 0.5 \mathrm{a}$ & $14.2 \pm 4.7 \mathrm{a}$ & $682 \pm 182 \mathrm{a}$ & $2,710 \pm 150 \mathrm{a}$ & $2,808 \pm 129 b$ & $4.0 \pm 3.6 \mathrm{a}$ & S & VT \\
\hline Celebration (M) & $4.9 \pm 0.1 \mathrm{a}$ & $7.9 \pm 1.5 \mathrm{a}$ & $361 \pm 32 \mathrm{a}$ & $2,747 \pm 314 a$ & $3,933 \pm 230 a b$ & $46.7 \pm 11.9 \mathrm{a}$ & MS & I \\
\hline Legacy $(\mathrm{M})$ & $4.7 \pm 0.3 \mathrm{a}$ & $7.3 \pm 3.5 \mathrm{a}$ & $621 \pm 218 a$ & $2,941 \pm 210 a$ & $4,138 \pm 268 a b$ & $42.5 \pm 12.6 \mathrm{a}$ & MS & I \\
\hline Morex (M) & $5.0 \pm 0.0 \mathrm{a}$ & $21.1 \pm 16.3 \mathrm{a}$ & $1,167 \pm 591 \mathrm{a}$ & $3,021 \pm 303 \mathrm{a}$ & $4,076 \pm 382 \mathrm{ab}$ & $35.2 \pm 2.8 \mathrm{a}$ & S & I \\
\hline Quest (M) & $4.7 \pm 0.3 \mathrm{a}$ & $4.9 \pm 0.5 \mathrm{a}$ & $721 \pm 421 \mathrm{a}$ & $3,243 \pm 326 a$ & $3,471 \pm 351 \mathrm{ab}$ & $9.3 \pm 13.0 \mathrm{a}$ & MR & $\mathrm{T}$ \\
\hline Tradition (M) & $4.5 \pm 0.5 \mathrm{a}$ & $6.6 \pm 1.3 \mathrm{a}$ & $782 \pm 162 \mathrm{a}$ & $3,202 \pm 631 \mathrm{a}$ & $3,947 \pm 312 a b$ & $35.0 \pm 24.7 \mathrm{a}$ & MS & I \\
\hline Mean & 4.7 & 10.4 & 707 & 3,306 & 3,942 & 23.0 & MS & MI \\
\hline$P>F$ & 0.6880 & 0.3752 & 0.1326 & 0.0624 & 0.0345 & 0.1304 & $\ldots$ & $\ldots$ \\
\hline $\mathrm{HSD}_{0.05}$ & Ns & ns & $\mathrm{ns}$ & ns & 878 & $\mathrm{~ns}$ & $\ldots$ & $\ldots$ \\
\hline
\end{tabular}

s Means and standard error of the mean for three replicates of each disease parameter and four replicates of grain parameters. Means followed by the same letter within a column did not differ significantly at $\alpha=0.05$ as determined by Tukey's honestly significant difference (HSD) test; ns $=$ not significant. Means of severity data for the root knot symptom were analyzed by the Kruskal-Wallis test and, when the Pearson's $\chi^{2}$ value for the experiment was significant at $\alpha<0.05$, cultivars were examined pairwise to determine which treatments differed significantly.

${ }^{\mathrm{t}}$ Root knot severity ratings for control (no-nematicide) treatment only, with a scale of $1=$ no evidence of damage, $2=1$ to $3 \mathrm{knots} / \mathrm{root}$ system, $3=3$ to $5 \mathrm{knots}, 4=$ $>5$ knots and $<20 \%$ reduction in plant height or root mass, and $5=>5$ knots and $>20 \%$ reduction in plant height or root mass. More than $98 \%$ of all cultivars exhibited the root knotting symptom on at least one root (e.g., the incidence was 98 to $100 \%$ ).

" Number of $H$. avenae white females produced per plant for the control (no-nematicide) treatment only; sampling was performed at about the time of anthesis.

v Number of $H$. avenae eggs per kilogram of soil for the control (no-nematicide) treatment only; extraction of cysts was performed from soil that was dry following harvest.

w Nematicide treatment included application of aldicarb (aldicarb at $4.2 \mathrm{~kg} / \mathrm{ha}$ ) in the seed row at the time of planting.

$x$ Percent increase in grain yield due to application of nematicide.

y Phenotypic resistance reaction: very resistant (VR; $\leq 1$ swollen female/plant), resistant (R; 1.1 to 3 ), moderately resistant (MR; 3.1 to 6), moderately susceptible (MS; 6.1 to 12 ), susceptible ( $; 12.1$ to 25 ), or very susceptible (VS; $>25$ ).

${ }^{\text {z }}$ Phenotypic tolerance reaction: very tolerant (VT; $<5 \%$ yield response to nematicide), tolerant ( $\mathrm{T} ; 5$ to $10 \%$ ), moderately tolerant (MT; 10 to $15 \%$ ), moderately intolerant (MI; 15 to $30 \%$ ), intolerant (I; 30 to $50 \%$ ), or very intolerant (VI; >50\%). 
Grain yield and test weight. Mean grain yield for all entries in each experiment were significantly greater during 2013 than during 2014 for the two-row feed and two-row malting barley $(P<$ $0.0001)$ and for the six-row barley $(P=0.0567)$. Treatment effects for cultivar and nematicide each differed significantly $(P$ $<0.01$ ) within each experiment. Mean grain yields were higher in nematicide-treated plots compared with the controls: 4,873 versus $4,496 \mathrm{~kg} / \mathrm{ha}\left(\mathrm{HSD}_{0.05}=148\right)$ for two-row feed barley, 4,784 versus $4,366 \mathrm{~kg} / \mathrm{ha}\left(\mathrm{HSD}_{0.05}=185\right)$ for two-row malt barley, and 4,847 versus $4,434 \mathrm{~kg} / \mathrm{ha}\left(\mathrm{HSD}_{0.05}=246\right)$ for six-row barley. The year-nematicide, cultivar-nematicide, and year-cultivar-nematicide interactions were not statistically significant for any of the three experiments. However, the year-cultivar interaction was significant $(P<0.01)$ for the two-row malt and six-row barley, and for the tworow feed barley $(P=0.0567)$. Grain yields for individual cultivars and for individual years in the three experiments are presented in Tables 1 to 3 . Grain yields for cultivars differ significantly $(P<0.05)$ within each nematicide treatment of the two-row feed barley and the two-row malt barley experiments during both years. For the six-row barley, grain yield did not differ among cultivars in the control treatment during either year, and differed significantly in the nematicide treatment only during 2014.

The percent increase in grain yield due to application of nematicide did not differ significantly in the two-row feed barley experiment (Table 1). For the two-row malt barley, the increase in grain yield was significant for the main effect of cultivar during 2014 but not during 2013 (Table 2). For the six-row barley, the main effects of year and cultivar were each significant. The year-cultivar interaction was significant $(P<0.01)$ for all three experiments.

Grain test weight differed significantly $(P<0.0001)$ between years for each of the three experiments. Mean test weights in the controls during 2013 and 2014 were 657 and $616 \mathrm{~g} /$ liter $\left(\mathrm{HSD}_{0.05}=6\right)$, respectively, for two-row feed barley; 635 and $584 \mathrm{~g} /$ liter $\left(\mathrm{HSD}_{0.05}=6\right)$, respectively, for two-row malt barley; and 592 and $556 \mathrm{~g} / \mathrm{liter}\left(\mathrm{HSD}_{0.05}=\right.$ 8 ), respectively, for six-row barley (data not presented). The main effect of cultivar also differed significantly $(P<0.05)$ for test weights in each experiment. Test weight data are not presented in the tables. The main effect of nematicide treatment differed significantly $(P=$ 0.03 ) for the two-row malt barley experiment but did not differ significantly for the other two experiments.

Balanced resistance and tolerance to $\boldsymbol{H}$. avenae. When data in our trials were grouped over 2 years, the percent increase in grain yield due to application of nematicide differed significantly among years only for the six-row barley experiments. The main effect of cultivar was significant for the two-row malt barley and the six-row barley experiments. The influence of year on numbers of white females produced on roots was significant for all three experiments, as was the main effect of cultivar for the two-row malt barley experiment. Recognizing the limits of the variability encountered, we used data grouped over 2 years to develop recommendations for managing barley fields that are infested by $H$. avenae (Table 4). This table identifies cultivars that could provide an acceptable balance of both resistance and tolerance, of resistance alone, of tolerance alone, or none of these traits.

Over the 2-year testing period, seven feed-type cultivars were ranked as having a balance of being at least moderately resistant and moderately tolerant (Table 4). These cultivars included the two-row barley Champion, Lenetah, Xena, 'Idagold II', and 'Transit' and the six-row barley Millennium and Goldeneye. No malting-type cultivar met these criteria. The two-row malt barley Odyssey was the only cultivar that exhibited resistance but not tolerance; it ranked as very resistant, with a mean of $<1$ swollen female/plant over the 2-year test period. This cultivar was not considered both resistant and tolerant because the tolerance rating of 15.5 did not strictly meet our maximum value of 15.0 to achieve that rating. Four cultivars also limited reproduction of $H$. avenae and were ranked as moderately resistant, including the food barley 'CDC Fibar', feed barley Steptoe, and the malt barley Legacy and Tradition, with means of 4.4, 5.3, 3.8, and 5.6 swollen females/plant, respectively. Eighteen barley cultivars (5 tworow feed types, 10 two-row malt types, 1 six-row feed type, and 2 six- row malt types) were ranked as being tolerant or very tolerant but not resistant or moderately resistant. Overall, $69 \%$ of the barley cultivars in these trials ( 31 of 45 entries) were at least moderately tolerant to H. avenae (Table 4).

\section{Discussion}

Small grain cultivars that are both resistant and tolerant to $H$. avenae are typically more profitable than cultivars that are susceptible and intolerant when planted into fields that are highly infested with this cereal cyst nematode (Fisher 1982). O'Brien and Fisher (1977) predicted that a combination of resistance and tolerance was more likely to be achieved in barley cultivars than in wheat or oat because barley is generally more tolerant of $H$. avenae than the other two crop species. We report the first-known screening trials to quantify both resistance and tolerance of barley to $H$. avenae in the United States.

We detected a balance of agronomically acceptable resistance plus tolerance in seven feed barley cultivars (five two-row and two sixrow types) but not in any malting barley. However, one malting type (Odyssey) nearly met this dual-trait criterion, in that it was very resistant and nearly moderately tolerant. Although neither the resistant cultivars nor the tolerant cultivars were always the highest-yielding cultivars in each trial, the long-term goals of producing a cultivar with these dual traits will be to simultaneously (i) minimize the potential yield suppression caused by the nematode in the current crop and (ii) reduce the postharvest density of eggs to reduce the economic risk for the next host crop that will be planted in that field. Six of the seven cultivars with balanced levels of resistance and tolerance (Xena, Champion, Goldeneye, Idagold II, Millennium, and Lenetah) produced grain yields in control plots that did not differ significantly from the highest-yielding cultivar in the experiment during either year. However, when compared with the highest-yielding cultivars in the two-row feed barley trial, Transit was categorized as resistant and tolerant but produced low yields each year. Transit, Julie, CDC Fibar, and CDC McGwire are hulless high $\beta$-glucan cultivars developed for human food, which are hulled, not as feed barley. Likewise, each of the four cultivars that were rated as susceptible but moderately resistant (CDC Fibar, Legacy, Steptoe, and Tradition) were not consistently among the highest-yielding cultivars within each of their experiments. Of the 18 cultivars that were rated as very tolerant or tolerant but not resistant, there were 7 cultivars that yielded among the top-ranked within their experiments both years, including the two-row feed types Baronesse, 'RWA 1758', Tetonia, and 'Vespa'; the two-row malt-types '2AB04-X001084-27' and '2Ab07-X031098-31'; and the six-row feed type 'Herald'. Cultivars such as 'Copeland' and '01AB9663' were among the highest yielding entries in their trials during 2013 but did not produce competitive yields during 2014, and the converse occurred for cultivars such as Merit 57, 'Overture', and Pinnacle.

We previously determined that wheat cultivars responded equally to $H$. avenae populations in Idaho, Oregon, and Washington (Smiley et al. 2011b, 2013). Therefore, it is anticipated that the resistance and tolerance traits identified for barley cultivars in these trials will also be applicable in $H$. avenae-infested fields elsewhere in the PNW.

Where resistance is the primary trait of interest to reduce the density of $H$. avenae in highly infested fields, we found that emphasis could be placed upon the seven dual-trait cultivars or upon the very resistant, resistant, and moderately resistant two-row food barley CDC Fibar, the two-row malt barley Odyssey, the six-row feed barley Steptoe, and the six-row malt barley Legacy and Tradition. Where tolerance is considered the primary trait of importance to achieve the maximum potential yield of feed barley or to achieve the highest malting quality, results of our study showed that emphasis could be placed either upon the dual-trait cultivars or upon production of 5 tolerant but not resistant two-row feed barley, 1 two-row feed barley, 10 six-row feed barley, and 2 six-row malt barley cultivars.

The origins of resistance traits detected in some cultivars examined in our trials are unknown. It appears that no North America accessions listed in the barley database of USDA-ARS-GRIN (2015) are designated as having resistance to $H$. avenae. Rivoal and Cook 
(1993) reviewed reports of unidentified sources of resistance to $H$. avenae being identified in locally selected barley in several countries. They also stated that "Undoubtedly, resistance genes have been dispersed...by the extensive collection and interchange of germplasm by plant breeders." It is likely that international exchanges of barley germplasm have led to the introgression of resistance genes into cultivars of current importance in the United States, and that these cultivars have not been assayed for resistance to $H$. avenae. The cultivars we report as resistant to $H$. avenae under field conditions should be verified as resistant using controlled-environment assays. Likewise, cultivars that are validated as resistant in subsequent investigations should be assayed using molecular markers (Barr et al. 1998; Chełkowski et al. 2003; Dayteg et al. 2008; Karakousis et al. 2003; Kretschmer et al. 1997; Seah et al. 1998) to identify the presence of one or more specific resistance genes or to provide provisional evidence for the occurrence of one or more new sources of resistance.

We ranked $69 \%$ of the 45 barley entries as being very tolerant, tolerant, or moderately tolerant $(11,11$, and 9 entries, respectively). Results of our research contribute to the understanding of differences in

Table 4. Summary of cultivar tolerance and resistance traits for data grouped over 2 years

\begin{tabular}{|c|c|c|c|c|c|}
\hline Cultivar ${ }^{u}$ & White females/plant ${ }^{\mathbf{v}}$ & Resistance ratingw $^{w}$ & Yield increase $(\%)^{x}$ & Tolerance rating ${ }^{\mathrm{y}}$ & $\mathbf{M R}+\mathbf{M T}^{\mathbf{z}}$ \\
\hline \multicolumn{6}{|l|}{ Two-row barley (F) } \\
\hline Julie & 6.2 & MS & 1.5 & VT & $\ldots$ \\
\hline RWA 1758 & 6.3 & MS & 4.7 & VT & $\ldots$ \\
\hline Tetonia & 13.1 & S & 4.6 & VT & $\ldots$ \\
\hline Vespa & 10.1 & MS & 3.0 & VT & $\ldots$ \\
\hline Baronesse & 6.2 & MS & 8.7 & $\mathrm{~T}$ & $\ldots$ \\
\hline Champion & 5.9 & MR & 7.2 & $\mathrm{~T}$ & $\mathrm{X}$ \\
\hline Lenetah & 2.6 & $\mathrm{R}$ & 9.8 & $\mathrm{~T}$ & $\mathrm{X}$ \\
\hline Xena & 3.4 & MR & 5.4 & $\mathrm{~T}$ & $\mathrm{X}$ \\
\hline 08ID2661 & 7.1 & MS & 12.4 & MT & $\ldots$ \\
\hline CDC McGwire & 8.6 & MS & 11.8 & MT & $\ldots$ \\
\hline Idagold II & 4.5 & MR & 10.6 & MT & $\mathrm{X}$ \\
\hline Spaulding & 14.5 & S & 12.1 & MT & $\ldots$ \\
\hline Transit & 4.5 & MR & 10.5 & MT & $\mathrm{X}$ \\
\hline 08ID1549 & 6.3 & MS & 16.1 & MI & $\ldots$ \\
\hline CDC Fibar (hulless) & 4.4 & MR & 18.2 & MI & $\ldots$ \\
\hline Clearwater & 7.3 & MS & 23.1 & MI & $\ldots$ \\
\hline \multicolumn{6}{|l|}{ Two-row barley (M) } \\
\hline 2Ab04-X001084-27 & 21.5 & $\mathrm{~S}$ & 3.0 & VT & $\ldots$ \\
\hline 2B05-0811 (B0811) & 12.2 & $\mathrm{~S}$ & 1.5 & VT & $\ldots$ \\
\hline Copeland & 19.4 & S & 4.4 & VT & $\ldots$ \\
\hline Merit & 15.3 & $S$ & 3.8 & VT & $\ldots$ \\
\hline Merem & 11.8 & MS & 5.6 & $\mathrm{~T}$ & $\ldots$ \\
\hline 2Ab07-X031098-31 & 11.4 & MS & 5.7 & $\mathrm{~T}$ & $\ldots$ \\
\hline LCS 1820 & 7.9 & MS & 8.9 & $\mathrm{~T}$ & $\ldots$ \\
\hline Merit 57 & 26.4 & VS & 6.5 & $\mathrm{~T}$ & $\ldots$ \\
\hline Overture & 17.1 & S & 9.1 & $\mathrm{~T}$ & $\ldots$ \\
\hline Pinnacle & 19.1 & S & 6.9 & $\mathrm{~T}$ & $\ldots$ \\
\hline Genie & 33.6 & VS & 10.7 & MT & $\ldots$ \\
\hline Harrington & 20.8 & S & 10.3 & MT & $\ldots$ \\
\hline Meredith & 13.8 & $S$ & 13.6 & MT & $\ldots$ \\
\hline ABI Voyager & 38.6 & VS & 19.0 & MI & $\ldots$ \\
\hline Conrad & 9.8 & MS & 16.8 & MI & $\ldots$ \\
\hline Hockett & 24.1 & $\mathrm{~S}$ & 16.6 & MI & $\ldots$ \\
\hline Metcalf & 16.5 & S & 16.8 & MI & $\ldots$ \\
\hline Odyssey & 0.9 & VR & 15.5 & MI & $\ldots$ \\
\hline B1202 & 23.8 & S & 30.2 & I & $\ldots$ \\
\hline \multicolumn{6}{|l|}{ Six-row barley } \\
\hline $01 \mathrm{Ab} 9663(\mathrm{M})$ & 7.8 & MS & 0.8 & VT & $\ldots$ \\
\hline Millennium (F) & 5.0 & MR & 2.6 & VT & $\mathrm{X}$ \\
\hline Quest (M) & 6.4 & MS & 3.0 & $\mathrm{VT}$ & $\ldots$ \\
\hline Herald (F) & 10.5 & MS & 5.6 & $\mathrm{~T}$ & $\ldots$ \\
\hline Goldeneye (F) & 5.5 & MR & 13.8 & MT & $\mathrm{X}$ \\
\hline Legacy (M) & 3.8 & MR & 27.2 & MI & $\ldots$ \\
\hline Morex (M) & 10.2 & MS & 17.6 & MI & $\cdots$ \\
\hline Steptoe $(\mathrm{F})$ & 5.3 & MR & 17.2 & MI & $\ldots$ \\
\hline Tradition (M) & 5.6 & MR & 20.8 & MI & $\ldots$ \\
\hline Celebration (M) & 6.4 & MS & 30.5 & I & $\ldots$ \\
\hline
\end{tabular}

${ }^{\mathrm{u}} \mathrm{F}=$ feed barley and $\mathrm{M}=$ malt barley.

v Number of $H$. avenae white females produced per plant for the control (no-nematicide) treatment.

${ }^{w}$ Cultivars were rated as very resistant (VR; $\leq 1$ swollen female/plant), resistant (R; 1.1 to 3 ), moderately resistant (MR; 3.1 to 6), moderately susceptible (MS; 6.1 to 12 ), susceptible ( $\mathrm{S} ; 12.1$ to 25 ), or very susceptible (VS; $>25$ ).

${ }^{x}$ Percent increase in grain yield due to application of nematicide.

y Tolerance ratings were very tolerant (VT; $<5 \%$ yield response to nematicide), tolerant (T; 5 to $10 \%$ ), moderately tolerant (MT; 10 to $15 \%$ ), moderately intolerant (MI; 15 to $30 \%$ ), intolerant (I; 30 to $50 \%$ ), or very intolerant (VI; $>50 \%$ ).

${ }^{\mathrm{z}}$ Cultivars that were neither resistant nor tolerant but which met a balanced criteria of being at least both moderately resistant ( $\leq 6 \%$ swollen females/plant) and moderately tolerant ( $\leq 15 \%$ yield increase with nematicide). 
tolerance that occur among North American spring barley cultivars but the origins of the tolerance traits we detected are unknown. Differences in tolerance to $H$. avenae are known to occur among cultivars of all small grain cereals, and it is generally accepted that there is an increasing order of yield damage caused by $H$. avenae on rye and winter barley, spring barley, winter wheat, spring wheat, winter oat, and spring oat (Andersson 1982; Fisher 1982). As such, a comparatively higher level of tolerance among spring barley than spring wheat or spring oat cultivars has been reported for many years. Attempts to characterize the mechanisms of tolerance have generally been associated with the timing of root development in these cereals. For instance, Kerry and Hague (1974) found that juveniles that invade seminal roots tend to mature into males and those that invade coronal roots tend to mature as females. In their study, in the United Kingdom, the nodal roots of spring barley contained few $H$. avenae individuals and nodal roots of winter wheat and winter oat were heavily invaded. The difference was attributed to development of nodal roots in spring barley during June and July, when few second-stage juveniles were present in soil, compared with the development of nodal roots for the winter-sown cereals during April, at a time when juveniles were most numerous in soil. Price and Hague (1981) determined that newly formed roots of oat in controlledenvironment studies became more heavily invaded by $H$. avenae compared with barley, wheat, or rye, suggesting differences in a trait such as attraction, penetration, or establishment of juveniles in the oat roots, or the emigration of juveniles from roots. Most studies have failed to identify any influence of leachates from various plant species on the stimulation of hatching of juveniles from cysts (Zheng et al. 1997). Other studies have shown that barley plants produce higher volumes and numbers of roots compared with other cereals (O'Brien and Fisher 1978; Price and Hague 1981; Stanton and Fisher 1988), which leads to a dilution effect of a given density of $H$. avenae in soil relative to the root mass available for penetration and establishment.

Although some barley cultivars were resistant to H. avenae, it was also clear that the incidence of root knotting on these resistant cultivars was similar to that for susceptible cultivars. This was attributed to the fact that second-stage juveniles penetrate epidermal cells behind the root cap (Price and Hague 1981; Price et al. 1983) and move intracellularly to the growth zone (Baldwin and Mundo-Ocampo 1991). The juveniles of both $H$. avenae and $H$. filipjevi successfully penetrate roots in equal numbers for resistant as well as susceptible cultivars (Andersen 1961; Cui et al. 2012; O'Brien and Fisher 1977; Ogbonnaya et al. 2001; Oka et al. 1997). After a succession of molts, the females reprogram root cells to induce the formation of specialized feeding cells (Hewezi et al. 2012). Cells of the syncytium develop but then deteriorate in resistant cultivars, causing death or suppressed reproductive capacity of the female associated with the deteriorated syncytium (Andres et al. 2001; Oka et al. 1997; Seah et al. 2000). Resistance is expressed by a reduced ability of the nematode to produce viable eggs. Therefore, resistant cultivars may reduce the density of $H$. avenae in soil but may still be sensitive to earlier root injury that leads to a reduction of plant growth and grain production. O'Brien and Fisher (1977) reported that similar numbers of larvae invaded seedling roots of both susceptible and resistant wheat cultivars but then the number of nematodes within roots and the number of sites of root-knotting continued to increase as susceptible plants became older, and the number of nematodes decreased but the sites of root-knotting remained the same as resistant plants became older. In our field studies, we were not able to detect differences in the amount of the root-knotting symptom in resistant and susceptible cultivars sampled at the time of plant anthesis. However, we did measure minor but significant reductions in the root-knotting symptom in aldicarb-treated compared with control plots. In contrast, the application of aldicarb greatly reduced the development of swollen white females on susceptible cultivars, and reduced the mean postharvest densities of $H$. avenae eggs by 54 to $78 \%$. Resistant cultivars also reduced the number of swollen white females when measured at the time of plant anthesis, and the postharvest density of $H$. avenae eggs per kilogram of soil. Postharvest densities of $H$. avenae in plots of resistant barley and in the aldicarb treatment were never reduced to a level that would be too low to affect the productivity of a subsequently planted intolerant cultivar of wheat or barley. A background density of the nematode was present because only a portion of $H$. avenae eggs hatch from cysts during a single season (Andersen 1961). A total of 40 to $90 \%$ of the eggs remaining within cysts typically hatch to produce the invasive juvenile stage each year (Andersen 1961; Andersen and Andersen 1970). Therefore, hatching from individual cysts is spread over many years and, in the soils where our trials were performed, a rotation away from cereals for only a single year is insufficient to adequately reduce the residual risk to subsequent cereal crops, even when they follow a resistant cultivar or soil that was previously treated with a nematicide such as aldicarb. These observations on barley were very similar to our observations of root knotting, development of gravid white females, and postharvest density of H. avenae eggs on spring wheat (Smiley et al. 2011a, 2013).

Aldicarb is a long-favored research tool for examining effects of H. avenae on spring cereals (Brown 1987; Meagher et al. 1978). Granules of aldicarb are banded with or below the seed at the time of planting (Smiley et al. 2005b, 2013; Taylor et al. 1999). Wu et al. (2007) recently reported that aldicarb was the best of four nematicides tested for reducing reproduction of $H$. avenae in wheat. This nematicide has a half-life of up to 5 weeks and is taken up by roots to reduce the Heterodera population early in the plant growth period. Aldicarb does not suppress effects of fungal root pathogens (Kimpinski and Johnson 1995; Kimpinski et al. 1987), typically results in improved grain yields for genotypes that are intolerant to the nematode, and, in our experience, had no influence on growth or yield of spring wheat where densities of plant-parasitic nematodes were very low (Smiley et al. 2005a). In this study, we found that aldicarb had a minimal but significant effect on the incidence and severity of the rootknotting symptom, greatly reduced the development of gravid white females, greatly reduced the postharvest density of $H$. avenae eggs in soil, and generally led to an increase of grain yield.

The initial density of $H$. avenae in the trial area was high during 2013 (22,176 eggs plus juveniles/kg of soil) and was variable for the three experiments during 2014: 3,516, 27,000, and 4,980 eggs plus juveniles $/ \mathrm{kg}$ of soil in the two-row feed, two-row malt, and six-row feed plus malt barley experiments, respectively. Each of these initial densities was high enough that yield suppression could be expected to occur during 2014 (Andersson 1982). Nearly all plants displayed the root-knotting symptom caused by $H$. avenae when roots were inspected at the time of anthesis during each year. This finding of root damage in all trials suggested that the nematode densities were sufficiently high in all trials to potentially suppress yields. Andersen (1960) reported that spring barley yields in Sweden were reduced by $16,17,21,40$, and $55 \%$ when the initial density of $H$. avenae was $1,000,2,500,5,000,10,000$, and 20,000 eggs plus juveniles $/ \mathrm{kg}$ of soil. However, it is also recognized that the damage threshold is highly variable and differs among plant cultivars, geographical areas, timing of the hatching interval relative to the planting date, climate, and variations of seasonal weather, soil moisture, soil temperature, and other edaphic factors (Andersen 1961; Handa et al. 1985; Li et al. 2012; Rivoal and Cook 1993; Yang et al. 2008).

During 2014, we observed a high incidence and severity of the root-knotting symptom but detected very few white females when roots were evaluated after heading and anthesis. This had not occurred during the previous 7 years, in which field trials on $H$. avenaeinfested fields were conducted at multiple locations throughout the PNW, including on fields of the same Idaho farm during the previous 4 years (2010 to 2013). The initial density of $H$. avenae eggs plus juveniles on the field where our 2014 trials were established was considered acceptable for assaying barley, as described above. The most susceptible cultivars in the two-row feed, two-row malt, and six-row barley trials produced means of 6,10 , and 21 cysts/plant during 2014, with initial densities of 3,516, 27,000, and 4,980 eggs plus juveniles $/ \mathrm{kg}$ of soil, respectively. In an adjacent trial with spring wheat during 2014, the most susceptible cultivar produced means of 51 cysts/plant with an initial density of 3,309 eggs plus juveniles/kg of soil. Andersen (1961) reported that the number 
of cysts produced per barley plant was $12,24,26$, and 33 cysts/plant at initial densities of 1,000, 2,500, 5,000, and 10,000 eggs plus juveniles $/ \mathrm{kg}$ of soil, respectively. Using that guideline, we would have anticipated means of at least 20 swollen females on roots of the most susceptible cultivars during 2014.

Our trials during 2014 were planted when the soil temperature at the depth of planting was $6.7^{\circ} \mathrm{C}$. Seedling emergence occurred about 3 weeks after planting. Tikhonova (1971) reported that $H$. avenae juveniles in the continental climate of the Bashkir region of central Russia emerged from cysts at temperatures of $5^{\circ} \mathrm{C}$ and above. Smiley et al. (2005b) reported that, during a single late winter and spring season in eastern Oregon, where the climate is only slightly milder than where our barley trials were performed in eastern Idaho, that secondstage juveniles of $H$. avenae began emerging from cysts very rapidly when average weekly air temperatures stabilized between 2 and $5^{\circ} \mathrm{C}$, and that peak densities of juveniles in soil occurred in March and April before declining sharply during May and becoming almost nondetectable in June. However, the specific chronological timing of the primary hatch can vary greatly over seasons (Andersen 1961).

$\mathrm{Li}$ et al. (2012) found $16^{\circ} \mathrm{C}$ to be the optimum temperature for penetration of roots by $H$. avenae, and 18 to $22^{\circ} \mathrm{C}$ to be the optimum temperature range for those juveniles to develop into gravid cysts. Although not evaluated, we assumed that juveniles had begun to move from cysts into the soil matrix at the approximate time we planted our trials (Kerry and Jenkinson 1976; Tikhonova 1971), that the density of juveniles in soils continued to increase sharply as the soil warmed during the 3 weeks between planting and seedling emergence (Smiley et al. 2005b), and that juveniles were capable of invading roots for at least 3 weeks after they emerged from cysts (Davies and Fisher 1976).

In our study during 2014, it is possible that the low numbers of swollen white females occurred because the primary hatching period occurred later than anticipated, which may have led to too few GGD for molting and egg production between the date of root invasion and crop maturation. Accumulation of GDD for nematode development $\left(8^{\circ} \mathrm{C}\right)$ following seedling emergence was much slower during 2014 than during 2013, causing us to dig roots for evaluations nearly 3 weeks later on a chronological scale during 2014 than during 2013; samplings were at 79 versus 59 days and 633 versus 619 GDD, respectively, after seedling emergence. It was unclear as to whether the soil temperature was more suboptimal for penetration of roots during 2014 compared with 2013, which could have delayed or reduced invasion and led to development of low numbers of gravid females.

The low numbers of swollen white females counted during 2014 could have also been an artifact of the sampling time if the juveniles invaded roots at low soil temperatures and developed sooner than we anticipated, causing them to turn brown and to become indistinguishable from the residual density of cysts formed on previous cereal crops. In a similar continental climate of south-central Russia, Tikhonova (1971) reported that juveniles required 35 to 45 days from the time of root penetration to sexual maturity. Our samplings at 59 and 79 days after planting were based upon our understanding of the greater importance of heat units than of chronological time for the development of swollen white females. Therefore, it is possible that our sampling during 2014 was too late to detect the greatest number of white females. We believe, however, that this possibility was unlikely because we conducted exploratory samplings in these trials on 12 June and 1 July, corresponding to 33 and 52 days or 211 and 343 GDD after seedling emergence. On both preliminary sampling dates, we were unable to detect more than a few swollen white females, leading us to perform our comprehensive sampling on 23 July, corresponding approximately to the equivalent number of GDD as used for our sampling during 2013.

During 2014, as compared with 2013 , very few $H$. avenae eggs were detected in soil following the harvests of even the most susceptible barley cultivars. It is unknown as to whether this observation of uniformly low densities was a result of marginal levels of initial inoculum, a temperature suboptimal for development of gravid females, a difference of soil sampling methods, an undetected anomaly associated with the procedures used for extraction of cysts and counting of eggs released from cysts, or some other reason. As discussed previously, the initial inoculum density is unlikely to have been responsible because the number of eggs plus juveniles at the time of planting was presumed to have been adequate for producing at least 20 cysts per plant on susceptible cultivars in each of the three barley trials. The low production of gravid females we counted in each trial during 2014 could have led to the low postharvest densities of $H$. avenae. In effect, it may have been possible for the low production of gravid females to result in a greater net loss of $H$. avenae inoculum due to few newly produced eggs in the newly formed cysts and the continued hatching or mortality of eggs plus juveniles from cysts produced 2 years earlier. There are large differences in number of eggs developed within individual cysts (Andersen 1961). The mean fecundity reported by Andersen (1961) was 200 to 250 eggs/cyst, a range from near 0 to 600 eggs/cyst, and with a trend for an increasing number of eggs per cyst when increasing numbers of cysts are produced. We used different soil sampling methods during the 2 years but that is unlikely to have led to the uniformly low $H$. avenae densities we detected. For sampling root-lesion nematodes, using the same equipment as for the current experiments, we reported similar results using 20 manually collected cores $(2.5 \mathrm{~cm}$ in diameter $)$ per plot and 2 mechanically collected cores $(5.1 \mathrm{~cm}$ in diameter) per plot (Smiley and Machado 2009). However, it is possible that the depth of sampling during 2014 was consistently closer to the target depth of $30 \mathrm{~cm}$ compared with the less-consistent depths that were possible when we sampled with hand probes during 2013. If true, the consistently deeper cores would have led to lower apparent densities of $H$. avenae because about $35 \%$ of cysts are found in the 5 - to $10-\mathrm{cm}$ depth interval and nearly $90 \%$ of cysts occur in the top $20 \mathrm{~cm}$ of noncultivated soil (Li et al. 2014; Xiang et al. 2013). We feel that it would be very unlikely for soil sampling methods alone to be responsible for the much lower magnitude of apparent egg counts during 2014 compared with 2013. Similarly, the extraction of cysts and counting of the released eggs is unlikely to have differed so greatly as to be responsible for the differences we encountered from year to year. The commercial laboratory that enumerated the numbers of $H$. avenae eggs for our trials is a high-throughput lab that used the same processing procedures and skilled staff each year.

In conclusion, we provided the first evaluations of tolerance and resistance of barley cultivars to $H$. avenae in the United States. Acceptable balances of resistance plus tolerance were detected in seven feed barley cultivars. One malting barley nearly met our criteria for this duality of traits. We also provided the first evidence of resistant but not tolerant cultivars in each of the four market classes of barley. Finally, we also reported the occurrence of barley cultivars in each market class that are tolerant but not resistant to $H$. avenae. Results of these studies provide the first basis for selecting barley cultivars and classes to improve production efficiency where barley is a desired crop on fields that are heavily infested by the cereal cyst nematode $H$. avenae.

\section{Acknowledgments}

We thank the Idaho Wheat Commission, Oregon Wheat Commission, Washington Wheat Commission, Idaho Agricultural Experiment Station, Oregon Agricultural Experiment Station, and United States Department of AgricultureAgricultural Research Service Root Disease and Biological Control Unit (at Pullman, WA) for funding; C. Jackson, T. Shelman, S. Arcibal, L. Beck, M. Carrillo, and E. Serna (University of Idaho, Aberdeen) and J. Gourlie, K. Rhinhart, P. Thorgersen, A. Hitzman, and N. Webster (Oregon State University, Pendleton) for technical assistance; Western Laboratories (Parma, ID) for providing discounted nematode testing fees; and D. Daw, St. Anthony, ID for providing land and crop management assistance.

\section{Literature Cited}

Andersen, K., and Andersen, S. 1970. Decrease of cereal cyst nematode infestation after growing resistant barley cultivars of grasses. Tidsskr. Planteavl 74: 559-565.

Andersen, S. 1960. Havreål problemer. Tolvmandsbladet 32:497-501.

Andersen, S. 1961. Resistens mod Havreål Heterodera avenae. Meddelelse nr. 68, Fra Den Kgl. Veterinaer-og Landbohojskoles afdeling for Landbrugets Plantekulture, Copenhagen, Denmark. 
Andersen, S., and Andersen, K. 1973. Linkage between marker genes on barley chromosome 2 and a gene for resistance to Heterodera avenae. Hereditas 73: 271-276.

Andersson, S. 1982. Population dynamics and control of Heterodera avenae-A review with some original results. EPPO Bull. 12:463-475.

Andres, M. F., Melillo, M. T., Delibes, A., Romero, M. D., and Bleve-Zacheo, T. 2001. Changes in wheat root enzymes correlated with resistance to cereal cyst nematodes. New Phytol. 152:343-354.

Baldwin, J. G., and Mundo-Ocampo, M. 1991. Heteroderinae, cyst- and non-cystforming nematodes. Pages 275-362 in: Manual of Agricultural Nematology. W. R. Nickle, ed. M. Dekker, New York.

Barr, A. R., Chalmers, K. J., Karakousis, A., Kretschmer, J. M., Manning, S., Lance, R. C. M., Lewis, J., Jeffries, S. P., and Langridge, P. 1998. RFLP mapping of a new cereal cyst nematode resistance locus in barley. Plant Breed. 117:185-187.

Brown, R. H. 1987. Control strategies in low-value crops. Pages 351-387 in: Principles and Practice of Nematode Control in Crops. R. H. Brown and B. R. Kerry, eds. Academic Press, Sydney, NSW, Australia.

Chełkowski, J., Tyrka, M., and Sobkiewicz, A. 2003. Resistance genes in barley (Hordeum vulgare L.) and their identification with molecular markers. J. Appl. Genet. 44:291-309.

Cook, R., and Evans, K. 1987. Resistance and tolerance. Pages 179-231 in: Principles and Practice of Nematode Control in Crops. R. H. Brown and B. R. Kerry, eds. Academic Press, Sydney, NSW, Australia.

Cotten, J. 1967. A comparison of cereal root eelworm resistant and susceptible spring barley genotypes at two sites. Ann. Appl. Biol. 59:407-413.

Cui, L., Gao, X., Wang, X. M., Jian, H., Tang, W. H., Li, H. L., and Li, H. J. 2012. Characteristics of interaction between wheat roots with different resistance and Heterodera filipjevi. Acta Agron. Sin. 38:1009-1017.

Davies, K. A., and Fisher, J. M. 1976. Duration of infectivity of second stage larvae of Heterodera avenae. Nematologica 22:163-168.

Dayteg, C., Rasmussen, M., Tuvesson, S., Merker, A., and Jahoor, A. 2008. Development of an ISSR-derived PCR marker linked to nematode resistance (Ha2) in spring barley. Plant Breed. 127:24-27.

Fisher, J. M. 1982. Problems with the use of resistance in wheat to the Australian pathotypes of Heterodera avenae. EPPO Bull. 12:417-421.

Fisher, J. M., and Hancock, T. W. 1991. Population dynamics of Heterodera avenae Woll. in South Australia. Aust. J. Agric. Res. 42:53-68.

Hajihassani, A., Tahna Maafi, Z., and Hajihasani, M. 2010. The life cycle of Heterodera filipjevi in winter wheat under microplot conditions in Iran. Nematol. Mediterr. 38:53-57.

Handa, D. K., Mathur, R. L., Mathur, B. N., and Yadav, B. D. 1985. Estimation of losses in barley due to cereal cyst nematode in sandy and sandy loam soils. Ind. J. Nematol. 15:163-166.

Hewezi, T., Maier, T. R., Nettleton, D., and Baum, T. J. 2012. The Arabidopsis microRNA396-GRF1/GRF3 regulatory module acts as a developmental regulator in the reprogramming of root cells during cyst nematode infection. Plant Physiol. 159:321-335.

Holgado, R., Andersson, S., Rowe, J., Clark, I., and Magnusson, C. 2009. Management strategies for cereal cyst nematodes Heterodera spp. in Norway. Pages 154-159 in: Cereal Cyst Nematodes: Status, Research and Outlook. I. T. Riley, J. M. Nicol, and A. A. Dababat, eds. CIMMYT, Ankara, Turkey.

Karakousis, A., Gustafson, J. P., Chalmers, K. J., Barr, A. R., and Langridge, P. 2003. A consensus map of barley integrating SSR, RFLP, and AFLP markers. Aust. J. Agric. Res. 54:1173-1185.

Kerry, B. R., and Hague, N. G. M. 1974. The invasion and development of the cereal cyst-nematode, Heterodera avenae in the roots of autumn- and springsown cereals. Ann. Appl. Biol. 78:319-330.

Kerry, B. R., and Jenkinson, S. C. 1976. Observations on emergence, survival and root invasion of second-stage larvae of the cereal cyst nematode, Heterodera avenae. Nematologica 22:467-474.

Kimpinski, J., and Johnson, H. W. 1995. Effects of aldicarb and fungicides on Pratylenchus penetrans populations, root rot and net blotch severity on barley. Phytoprotection 76:9-16.

Kimpinski, J., Johnson, H. W., and Martin, R. A. 1987. Influence of aldicarb on root lesion nematodes, leaf disease and root rot in wheat and barley. Plant Pathol. 36:333-338.

Kretschmer, J. M., Chalmers, K. J., Manning, S., Karakousis, A., Barr, A. R., Islam, A. K. M. R., Logue, S. J., Choe, Y. W., Barker, S. J., Lance, R. C. M., and Langridge, P. 1997. RFLP mapping of the Ha 2 cereal cyst nematode resistance gene in barley. Theor. Appl. Genet. 94:1060-1064.

Li, X. H., Ma, J., and Chen, S. L. 2012. Effect of temperature on the penetration and development of Heterodera avenae. J. Triticeae Crops 32:977-981

Li, X. H., Ma, J., Chen, S. L., Gao, B., and Wang, R. Y. 2014. Vertical and horizontal distribution of Heterodera avenae in the field. Plant Prot. 40: 140-143.

Marshall, J., Jackson, C., Shelman, T., Beck, L., and O'Brien, K. 2014. 2013 Small Grains Report. Univ. Idaho Res. Bull. 182. Online publication. http://www. uidaho.edu/extension/cereals/scseidaho/sgr

Meagher, J. W., Brown, R. H., and Rovira, A. D. 1978. The effect of cereal cyst nematode (Heterodera avenae) and Rhizoctonia solani on the growth and yield of wheat. Aust. J. Agric. Res. 29:1127-1137.
Moseman, J. G. 1972. Report on genes for resistance to pests. Barley Genet. Newsl. 2:145-146.

Nielsen, C. H. 1982. Heredity of Heterodera avenae resistance originating from two barley cultivars and one spring wheat cultivar. EPPO Bull. 12: 457-461.

O'Brien, P. C., and Fisher, J. M. 1974. Resistance within wheat, barley and oat cultivars to Heterodera avenae in South Australia. Aust. J. Exp. Agric. Anim. Husb. 14:399-404.

O'Brien, P. C., and Fisher, J. M. 1977. Development of Heterodera avenae on resistant wheat and barley cultivars. Nematologica 23:390-397.

O'Brien, P. C., and Fisher, J. M. 1978. Factors influencing the number of larvae of Heterodera avenae within susceptible wheat and barley seedlings. Nematologica 24:295-304

Ogbonnaya, F. C., Subrahmanyam, N. C., Moullet, O., de Majnik, J., Eagles, H. A., Brown, J. S., Eastwood, R. F., Kollmorgan, J., Appels, R., and Lagudah, E. S. 2001. Diagnostic DNA markers for cereal cyst nematode resistance in bread wheat. Aust. J. Agric. Res. 52:1367-1374.

Oka, Y., Chet, I., and Speigel, Y. 1997. Accumulation of lectins in cereal roots invaded by the cereal cyst nematode Heterodera avenae. Physiol. Mol. Plant Pathol. 51:333-345.

Price, N. S., Clarkson, D. T., and Hague, N. G. M. 1983. Effect of the invasion by cereal cyst nematode (Heterodera avenae) on the growth and development of the seminal roots of oats and barley. Plant Pathol. 32:377-383.

Price, N. S., and Hague, N. G. M. 1981. The invasion of root tips of cereals by the cereal cyst nematode Heterodera avenae. Ann. Appl. Biol. 99:301-306.

Rivoal, R., and Cook, R. 1993. Nematode pests of cereals. Pages 259-303 in: Plant Parasitic Nematodes in Temperate Agriculture. K. Evans, D. L. Trudgill, and J. M. Webster, eds. CAB International, Wallingford, UK

Rivoal, R., and Sarr, E. 1987. Field experiments on Heterodera avenae in France and implications for winter wheat performance. Nematologica 33: 460-479.

Seah, S., Miller, C., Sivasithamparam, K., and Lagudah, E. S. 2000. Root responses to cereal cyst nematode (Heterodera avenae) in hosts with different resistance genes. New Phytol. 146:527-533.

Seah, S., Sivasithamparam, K., Karakousis, A., and Lagudah, E. S. 1998. Cloning and characterisation of a family of disease resistance gene analogs from wheat and barley. Theor. Appl. Genet. 97:937-945

Smiley, R. W. 2009. Occurrence, distribution and control of Heterodera avenae and $H$. filipjevi in the western USA. Pages 35-40 in: Cereal Cyst Nematodes: Status, Research and Outlook. I. T. Riley, J. M. Nicol, and A. A. Dababat, eds. CIMMYT, Ankara, Turkey.

Smiley, R. W., Gourlie, J. A., Rhinhart, K. E. L., Marshall, J. M., Anderson, M. D. and Yan, G. P. 2012. Influence of nematicides and fungicides on spring wheat in fields infested with soilborne pathogens. Plant Dis. 96:1537-1547.

Smiley, R. W., Ingham, R. E., Uddin, W., and Cook, G. H. 1994. Crop sequences for managing cereal cyst nematode and fungal populations of winter wheat. Plant Dis. 78:1142-1149.

Smiley, R. W., and Machado, S. 2009. Pratylenchus neglectus reduced yield of winter wheat in dryland cropping systems. Plant Dis. 93:263-271.

Smiley, R. W., Marshall, J. M., Gourlie, J. A., Paulitz, T. C., Kandel, S. L., Pumphrey, M. O., Garland-Campbell, K., Yan, G. P., Anderson, M. D., Flowers, M. D., and Jackson, C. A. 2013. Spring wheat tolerance and resistance to Heterodera avenae in the Pacific Northwest. Plant Dis. 97: 590-600.

Smiley, R. W., Marshall, J. M., and Yan, G. P. 2011a. Effect of foliarly-applied spirotetramat on reproduction of Heterodera avenae on wheat roots. Plan Dis. 95:983-989.

Smiley, R. W., Whittaker, R. G., Gourlie, J. A., and Easley, S. A. 2005a Pratylenchus thornei associated with reduced wheat yield in Oregon. J. Nematol. 37:45-54.

Smiley, R. W., Whittaker, R. G., Gourlie, J. A., Easley, S. A., and Ingham, R. E. 2005b. Plant-parasitic nematodes associated with reduced wheat yield in Oregon: Heterodera avenae. J. Nematol. 37:297-307.

Smiley, R. W., Yan, G. P., and Pinkerton, J. N. 2011b. Resistance of wheat, barley and oat to Heterodera avenae in the Pacific Northwest USA. Nematology 13: 539-552.

Stanton, J. M., and Fisher, J. M. 1988. Factors of early growth associated with tolerance of wheat to Heterodera avenae. Nematologica 34:188-197.

Taylor, S. P., Vanstone, V. A., Ware, A. H., McKay, A. C., Szot, D., and Russ, M. H. 1999. Measuring yield loss in cereals caused by root lesion nematodes (Pratylenchus neglectus and P. thornei) with and without nematicides. Aust. J. Agric. Res. 50:617-622.

Tikhonova, L. V. 1971. Ontogenesis of Heterodera avenae in cereal crops. Byull Vses. Inst. Gel'mint. K. I. Skry-abina. 6:105-114.

Tikhonova, L. V., Ten'kovtseva, E. S., and Airapetyan, V. A. 1975. Data on the organisation of crop rotation schemes aimed at a drastic reduction in the numbers of Heterodera avenae. Byull. Vses. Inst. Gel'mint. K. I. Skry-abina. 15:102-108.

USDA-ARS-GRIN. 2015. United States Department of Agriculture-Agricultural Research Service, Germplasm Resources Information Network. Online publication. http://www.ars-grin.gov/npgs/

USDA-NASS. 2013. Online publication. United States Department of Agriculture-National Agricultural Statistics Service. http://www.nass.usda.gov 
Valocká, B., Sabová, M., and Lišková, M. 1994. Response of some winter wheat and spring barley cultivars to Heterodera avenae pathotype Ha 12 . Helminthologia 31:155-158.

Williams, T. D. 1970. Barley segregates resistant and susceptible to the cereal cystnematode (Heterodera avenae Woll.). Ann. Appl. Biol. 66:339-346.

Wilson, R. E., Hollamby, G. J., and Bayraktar, A. 1983. Selecting for high yield potential in wheat with tolerance to cereal cyst nematode. Aust. Field Crops Newsl. 18:21-25.

Wu, X. J., Yang, W. X., Sun, B. J., Xing, X. P., Wang, Z. Y., Li, H. L., and Yuan, H. X. 2007. Effect of different nematicides on the controlling cereal cyst nematode and wheat growth. J. Henan Agric. Sci. 5:57-60.

Xiang, G. L., Song, Z. Q., Liang, X. D., Hu, X. B., Qi, Z. R., Wang, X., and Li, H. M. 2013. Life cycle and vertical distribution of Heterodera avenae on wheat in Peixian, Jiangsu Province, China. J. Triticeae Crops 33:789-794.
Yan, G. P., and Smiley, R. W. 2010. Distinguishing Heterodera filipjevi and $H$. avenae using polymerase chain reaction-restriction fragment length polymorphism and cyst morphology. Phytopathology 100:216-224.

Yan, G. P., Smiley, R. W., Okubara, P. A., and Skantar, A. M. 2013. Speciesspecific PCR assays for differentiating Heterodera filipjevi and $H$. avenae. Plant Dis. 97:1611-1619.

Yang, W. X., Yuan, H. X., Xing, X. P., Wang, Z. Y., and Li, H. L. 2008. Effect of soil constituents and soil moisture on cereal cyst nematode. Henan Sci. 26:672-675.

Yuan, H. X., Yan, H. T., Sun, B. J., Xing, X. P., and Li, H. L. 2014. Infection dynamics of two species of cereal cyst nematode in Zhengzhou, Henan Province. Acta Phytopathol. Sin. 44:74-79.

Zheng, J. W., Cheng, H. R., and Fang, Z. D. 1997. Hatching characteristics of cereal cyst nematode, Heterodera avenae in Shanxi, China. J. Zhejiang Agric. Univ. 26:667-671 